\title{
Some Degenerate Elliptic Systems and Applications to Cusped Plates
}

\author{
George Jaiani
}

Bert-Wolfgang Schulze

December 22, 2004

\begin{abstract}
The tension-compression vibration of an elastic cusped plate is studied under all the reasonable boundary conditions at the cusped edge, while at the noncusped edge displacements and at the upper and lower faces of the plate stresses are given.
\end{abstract}

Mathematics Subject Classification 2000. Primary 74K20; Secondary 35J70

Key words and phrases. Casped plates, vibration, degenerate elliptic systems, weighted spaces, Hardy's inequality, Korn's weighted inequality.

\section{Contents}

Introduction 1

1 The Resolving System of Degenerate Equations 2

2 Weighted Function Spaces 5

3 Hardy's Inequality $\quad 15$

4 Korn's Weighted Inequality 17

5 Weighted Boundary Value Problems $\quad 19$

6 Existence and Uniqueness Theorems 22

7 Some General Remarks $\quad 30$

$\begin{array}{ll}\text { References } & 31\end{array}$

\section{Introduction}

The present paper studies elastic plates the thickness of which may vanish on a part of the plate projection boundary, i.e., so called cusped plates. The tension- 
compression vibration of cusped plates is considered within the framework of the $N=1$ approximation of I. Vekua's hierarchical models. For the corresponding degenerate static system when the thickness is given by $2 h=x_{2}^{\kappa}, \kappa=$ const $>$ $0, x_{2} \geq 0$, the homogeneous Dirichlet problem was studied in G. Devdariani, G. Jaiani, S. Kharibegashvili, D. Natroshvili, 2000 and G. Devdariani, 2001. Now, we investigate the vibration system (if the vibration frequency is equal to zero, we get the static system) under all admissible nonhomogeneous Dirichlet, weighted Neumann, and mixed boundary conditions (BCs) when the thickness satisfies the unilateral condition $2 h\left(x_{1}, x_{2}\right) \leq h_{\kappa} x_{2}^{\kappa}, h_{\kappa}=$ const $>0, \kappa=$ const $\geq 0, x_{2} \geq 0$. The bending vibration problem can be investigated in an analogous manner.

The paper is organized as follows. In Section 1 we establish the vibration system in the first approximation and formulate some auxiliary facts. In Section 2 we introduce and investigate some necessary weighted Sobolev spaces. Section 3 deals with the proof of Hardy's inequality in weighted Sobolev spaces. Section 4 is devoted to Korn's weighted inequaltiy. In Section 5 we formulate the admissible boundary value problems (BVPs). In Section 6 we prove existence and uniqueness theorems. Finally, Section 7 is devoted to some general comments. Historically, the first models of elastic deformable bodies were 1D and 2D models, and then the 3D linear model of elastic bodies. In the middle of the XX century attempts were made, on the one hand to refine classical 1D and 2D models and, on the other hand, to obtain them from the 3D linear model pre-supposing the displacement to be polynomial in the thickness variable, transversal to the middle-surface. Such 2D models are known as hierarchical models. First achievements in this direction can be found in I. Vekua, 1955, 1965, 1985. Existence and uniqueness theorems in Sobolev spaces for I. Vekua's hierarchical models in the static case were proved by D. Gordeziani, 1974a. He also estimated the rate of approximation for these models in $C^{k}$ spaces, cf. D. Gordeziani 1974b. These investigations found their logical completion in M. Avalishvili, D. Gordeziani, 2003. In this direction Ch. Schwab's 1996 work is also remarkable. Various aspects of I. Vekua's models were studied by T. Vashakmadze 1999, T. Meunargia, 1998, V. Zhgenti, 1991, I. Khoma, 1986, V. Guliaev, V. Baganov, P. Lizunov, 1978, A. Khvoles, 1971, etc. A new stage for models in variational form began with the work of M. Vogelius, I. Babuška, 1981a,b. In the finite element framework the idea of hierarchical models has been first formulated by B. Szabó, G. Sahrmann, 1988, for isotropic domains, mathematically investigated by I. Babuška, L. Li, 1991, 1992a,b, and generalized to laminated composites by I. Babuška, B. Szabó, R. Actis, 1992, R. Actis, B. Szabó, Ch. Schwab, 1999. More details may be found in a survey paper of M. Dauge, E. Faou, Z. Yosibash 2004.

\section{The Resolving System of Degenerate Equations}

In the $N=1$ model (approximation) of I. Vekua's hierarchical models of symmetric prismatic shells, i.e., plates of variable thickness $2 h\left(x_{1}, x_{2}\right) \in C^{1}(\omega) \cap C(\bar{\omega})$ the tension-compression vibration system has the following form:

$$
L u=f \quad \text { in } \quad \omega
$$


where $\omega$ is a bounded open set in $\mathbb{R}^{2}$ with Lipschitz boundary (specified below),

$$
\begin{aligned}
& L:=\left(L_{1}, L_{2}, 3 L_{3}\right), u:=\left(u_{1}, u_{2}, v_{3}\right), f:=\left(f_{1}, f_{2}, f_{3}\right):=-\left(X_{1}, X_{2}, 3 X_{3}\right), \\
& L_{1} u \quad:=(\lambda+2 \mu)\left(h u_{1,1}\right)_{, 1}+\mu\left(h u_{1,2}\right)_{, 2}+\lambda\left(h u_{2,2}\right)_{, 1} \\
& +\quad \mu\left(h u_{2,1}\right)_{, 2}+3 \lambda\left(h v_{3}\right)_{, 1}+c^{2} p h u_{1}=-X_{1}, \\
& L_{2} u:=\mu\left(h u_{2,1}\right)_{, 1}+(\lambda+2 \mu)\left(h u_{2,2}\right)_{, 2}+\mu\left(h u_{1,2}\right)_{, 1}+\lambda\left(h u_{1,1}\right)_{, 2} \\
& +\quad 3 \lambda\left(h v_{3}\right)_{, 2}+c^{2} p h u_{2}=-X_{2}, \\
& L_{3}(u):=\mu\left(h^{3} v_{3,1}\right)_{, 1}+\mu\left(h^{3} v_{3,2}\right)_{, 2}-\lambda h u_{1,1}-\lambda h u_{2,2} \\
& -\quad 3(\lambda+2 \mu) h v_{3}+c^{2} p h^{3} v_{3}=-h X_{3}, \quad\left(x_{1}, x_{2}\right) \in \omega .
\end{aligned}
$$

Here $u_{1}:=\stackrel{1}{v}_{10}, u_{2}:=\stackrel{1}{v}_{20}$ are so called zero weighted moments, and $v_{3}:=\stackrel{1}{v}_{31}$ is the so called first weighted moment (see Vekua 1965) of the corresponding components of $U\left(x_{1}, x_{2}, x_{3}\right):=\left(U_{1}, U_{2}, U_{3}\right)$ (note that in our case the displacement vector has the form $\left.U\left(x_{1}, x_{2}, x_{3}, t\right)=e^{i c t} U\left(x_{1}, x_{2}, x_{3}\right)\right) ; \lambda>0$ and $\mu>0$ are the Lamé constants, $X_{\alpha}, \alpha=1,2$ are the sums of some combinations of the $\alpha$-th component of the surface forces acting on the plate faces and of the zero moments of the $\alpha$-th component of the volume forces; $X_{3}$ is the sum of a combination of the third components of the surface forces acting on the plate faces and of the first moment of the third component of the volume forces, indices after 'comma' mean differentiation with respect to the corresponding variables, $p$ is the plate density, $c$ is the vibration frequency, and $\omega$ is a projection on the plane $x_{3}=0$ of the plate $\bar{\Omega}$ :

$$
\Omega:=\left\{\left(x_{1}, x_{2}, x_{3}\right) \in R^{3}:\left(x_{1}, x_{2}\right) \in \omega,-h\left(x_{1}, x_{2}\right)<x_{3}<h\left(x_{1}, x_{2}\right)\right\} .
$$

If $c=0$, from (1.1) we get the system corresponding to the static case. We suppose that $\omega$ has a Lipschitz boundary $\partial \omega=\bar{\gamma}_{0} \cup \bar{\gamma}$, where $\bar{\gamma}_{0}$ is a segment of the axis $x_{1}$, and $\gamma$ lies in the upper half-plane $x_{2}>0$. Let

$$
2 h\left(x_{1}, x_{2}\right)>0 \quad \text { if } \quad\left(x_{1}, x_{2}\right) \in \omega \cup \gamma
$$

and

$$
2 h\left(x_{1}, 0\right) \geq 0 \quad \text { if } \quad\left(x_{1}, 0\right) \in \bar{\gamma}_{0} .
$$

When $h\left(x_{1}, 0\right)=0$, the plate is called a cusped one. Note that in the latter case, on the one hand, a 3D domain $\Omega$ occupied by the plate will be, in general, a domain with a non-Lipschitz boundary, on the other hand, the elliptic in $\omega$ system (1.1) will have an order degeneration on $\gamma_{0}$.

Let

$$
u, u^{*} \in C^{2}(\omega) \cap C^{1}(\bar{\omega}), \quad u^{*}:=\left(u_{1}^{*}, u_{2}^{*}, u_{3}^{*}\right) .
$$

Evidently, after multiplication by $u^{*}$ and integration by parts from (1.1) we obtain, that

$$
\int_{\omega} L u \cdot u^{*} d \omega=-\int_{\omega} B_{c}\left(u, u^{*}\right) d \omega-\int_{\partial \omega} T_{n} u \cdot u^{*} d \partial \omega=\int_{\omega} f \cdot u^{*} d \omega,
$$


where $n:=\left(n_{1}, n_{2}, n_{3}\right)$ is the inward normal, $T_{n}:=\left(X_{n 10}, X_{n 20}, 3 h X_{n 31}\right)$ with

$$
\begin{aligned}
X_{n 10} u= & \sum_{j=1}^{3} \stackrel{1}{\sigma}_{1 j 0} n_{j}=\sum_{\beta=1}^{2} \stackrel{1}{\sigma}_{1 \beta 0} n_{\beta} \\
= & h\left\{\left[(\lambda+2 \mu) u_{1,1}+\lambda u_{2,2}+3 \lambda v_{3}\right] n_{1}+\mu\left(u_{1,2}+u_{2,1}\right) n_{2}\right\}, \\
X_{n 20} u= & \sum_{\beta=1}^{2} \stackrel{1}{\sigma}_{2 \beta 0} n_{\beta} \\
= & h\left\{\mu\left(u_{2,1}+u_{1,2}\right) n_{1}+\left[(\lambda+2 \mu) u_{2,2}+\lambda u_{1,1}+3 \lambda v_{3}\right] n_{2}\right\}, \\
& h X_{n 31} u=\sum_{\beta=1}^{2} h^{1} \sigma_{3 \beta 1} n_{\beta}=h^{3} \mu \sum_{\beta=1}^{2} v_{3, \beta} n_{\beta},
\end{aligned}
$$

$\stackrel{1}{\sigma}_{\alpha \beta 0}$ and $\stackrel{1}{\sigma}_{3 \beta 1}, \alpha, \beta=1,2$, denote in the $N=1$ approximation the zero and first moments, respectively, of the corresponding components of the 3D stress tensor $\sigma_{i j}$, $i, j=1,2,3$,

$$
\begin{aligned}
B_{c}\left(u, u^{*}\right):= & (\lambda+2 \mu) h u_{1,1} u_{1,1}^{*}+\mu h u_{1,2} u_{1,2}^{*}+\lambda h u_{2,2} u_{1,1}^{*} \\
& +\mu h u_{2,1} u_{1,2}^{*}+3 \lambda h v_{3} u_{1,1}^{*}-c^{2} p h u_{1} u_{1}^{*}+\mu h u_{2,1} u_{2,1}^{*} \\
& +(\lambda+2 \mu) h u_{2,2} u_{2,2}^{*}+\mu h u_{1,2} u_{2,1}^{*}+\lambda h u_{1,1} u_{2,2}^{*}+3 \lambda h v_{3} u_{2,2}^{*} \\
& -c^{2} p h u_{2} u_{2}^{*}+3 \mu h^{3} v_{3,1} v_{3,1}^{*}+3 \mu h^{3} v_{3,2} v_{3,2}^{*}+3 \lambda h u_{1,1} v_{3}^{*}+3 \lambda h u_{2,2} v_{3}^{*} \\
& +9(\lambda+2 \mu) h v_{3} v_{3}^{*}-3 c^{2} p h^{3} v_{3} v_{3}^{*} \\
= & \lambda h\left(u_{1,1}+u_{2,2}+3 v_{3}\right)\left(u_{1,1}^{*}+u_{2,2}^{*}+3 v_{3}^{*}\right) \\
& +\mu h\left[2 u_{1,1} u_{1,1}^{*}+2 u_{2,2} u_{2,2}^{*}+\left(u_{2,1}+u_{1,2}\right)\left(u_{2,1}^{*}+u_{1,2}^{*}\right)+18 v_{3} v_{3}^{*}\right] \\
& +3 \mu h^{3}\left(v_{3,1} v_{3,1}^{*}+v_{3,2} v_{3,2}^{*}\right)-c^{2} p\left[h\left(u_{1} u_{1}^{*}+u_{2} u_{2}^{*}\right)+3 h^{3} v_{3} v_{3}^{*}\right] \\
=: & B\left(u, u^{*}\right)-c^{2} p\left[h\left(u_{1} u_{1}^{*}+u_{2} u_{2}^{*}\right)+3 h^{3} v_{3} v_{3}^{*}\right] .
\end{aligned}
$$

Note that the bilinear operators $B_{c}\left(u, u^{*}\right)$ and $B\left(u, u^{*}\right)$ correspond to the vibration and static cases, respectively. Obviously,

$$
\begin{aligned}
B(u, u)= & \lambda h\left(u_{1,1}+u_{2,2}+3 v_{3}\right)^{2} \\
& +\mu h\left[2\left(u_{1,1}\right)^{2}+2\left(u_{2,2}\right)^{2}+\left(u_{2,1}+u_{1,2}\right)^{2}+18\left(v_{3}\right)^{2}\right] \\
& +3 \mu h^{3}\left[\left(v_{3,1}\right)^{2}+\left(v_{3,2}\right)^{2}\right] \geq 0 \text { in } \omega .
\end{aligned}
$$

Non-negativeness of $B_{c}(u, u)$ causes a restriction on the vibration frequency $c$. This restriction is connected with the minimal eigenvalue problem for the system (1.1) and will be clarified below. 
If we consider boundary value problems (BVPs) for the system (1.1) with homogeneous BCs when on $\partial \omega$ either

$$
u=0,
$$

or

$$
T_{n} u=0,
$$

or

$$
u_{\alpha}=0, \alpha=1,2 ; \quad h X_{n 31}=0
$$

or

$$
X_{n \alpha 0}=0, \alpha=1,2 ; \quad v_{3}=0,
$$

or

$$
u_{1}=0, X_{n 20}=0, \quad h X_{n 31}=0,
$$

or

$$
X_{n 10}=0, u_{2}=0, \quad h X_{n 31}=0,
$$

or

$$
u_{1}=0, X_{n 20}=0, v_{3}=0
$$

or

$$
X_{n 10}=0, u_{2}=0, v_{3}=0,
$$

or on different parts of $\partial \omega$ different BCs (1.12) -(1.19) are given, then in (1.6) the integral along $\partial \omega$ will disappear and we obtain

$$
\int_{\omega} B_{c}\left(u, u^{*}\right) d \omega=-\int_{\omega} f \cdot u^{*} d \omega .
$$

Equality (1.20) will play crucial role in the definition of weak solutions of the above BVPs for the systems (1.1). It is remarkable, that as it will be shown below, $u_{1}, u_{2}$, and $v_{3}$ cannot be, in general prescribed on $\gamma_{0}$. The admissibility of Dirichlet conditions for $u_{1}, u_{2}$, and $v_{3}$ depends on the order of degeneration of the system (1.1) or, in other words, on the geometry of the plate sharpening. For instance, when the plate as $3 \mathrm{D}$ body has either a cuspidal edge (i.e., $\partial \Omega$ is non-Lipschitz boundary) or an angular edge, then $u_{1}, u_{2}$ and $v_{3}$ cannot be given on $\gamma_{0}$.

\section{Weighted Function Spaces}

Let us introduce some weighted spaces.

\section{Definition 2.1 By}

$$
W_{2}^{1}\left(\omega, \begin{array}{c}
\rho_{0} \\
\rho_{1}
\end{array}\right)
$$

we denote a set of all measurable functions $\varphi$ defined on $\omega$ which have on $\omega$ generalized (regular distributional) derivatives

$$
\partial_{x_{1}, x_{2}}^{\left(\alpha_{1}, \alpha_{2}\right)} \varphi \quad \text { for } \quad \alpha_{1}+\alpha_{2} \leq 1, \quad \alpha_{1}, \alpha_{2} \in\{0,1\}, \quad \partial_{x_{1}, x_{2}}^{(0,0)} \varphi \equiv \varphi,
$$


such that

$$
\int_{\omega}\left|\partial_{x_{1}, x_{2}}^{\left(\alpha_{1}, \alpha_{2}\right)} \varphi\right|^{2} \rho_{\alpha_{1}+\alpha_{2}} d \omega<+\infty
$$

where $\rho_{0}$ and $\rho_{1}$ are measurable, a.e. positive and finite on $\omega$ functions.

The space (2.1) is endowed with the norm

$$
\|\varphi\|_{W_{2}^{1}\left(\omega, \rho_{\rho_{1}}\right)}^{2}:=\int_{\omega}\left\{\rho_{0} \varphi^{2}+\rho_{1}\left[(\varphi, 1)^{2}+(\varphi, 2)^{2}\right]\right\} d \omega
$$

and the scalar product

$$
(\varphi, \psi)_{W_{2}^{1}\left(\omega, \rho_{\rho_{1}}\right)}:=\int_{\omega}\left[\rho_{0} \varphi \psi+\rho_{1}\left(\varphi_{, 1} \psi_{, 1}+\varphi_{, 2} \psi_{, 2}\right] d \omega .\right.
$$

Definition $2.2 W_{2}^{1}(\omega, \rho):=W_{2}^{1}\left(\omega, \begin{array}{l}\rho \\ \rho\end{array}\right)$.

According to Definitions 2.1, 2.2 we have the following sets

$$
\begin{aligned}
& W_{2}^{1}\left(\omega, \begin{array}{l}
1 \\
h
\end{array}\right), W_{2}^{1}(\omega, h), W_{2}^{1}\left(\omega, \begin{array}{c}
h \\
h^{3}
\end{array}\right), W_{2}^{1}\left(\omega, \begin{array}{l}
1 \\
x_{2}^{\kappa}
\end{array}\right), W_{2}^{1}\left(\omega, x_{2}^{\kappa}\right), \\
& W_{2}^{1}\left(\omega, \begin{array}{l}
x_{2}^{\kappa} \\
x_{2}^{3 \kappa}
\end{array}\right), W_{2}^{1}\left(\omega, x_{2}^{3 \kappa}\right), W_{2}^{1}\left(\omega, \begin{array}{l}
x_{2}^{\kappa-2} \\
x_{2}^{\kappa}
\end{array}\right), W_{2}^{1}\left(\omega, \begin{array}{l}
x_{2}^{\kappa-2} \\
h
\end{array}\right), \\
& W_{2}^{1}\left(\omega, \begin{array}{l}
1 \\
d^{\kappa}
\end{array}\right), W_{2}^{1}\left(\omega, d^{\kappa}\right), W_{2}^{1}\left(\omega, d^{3 \kappa}\right),
\end{aligned}
$$

where

$$
\kappa=\text { const }>0
$$

and

$$
d\left(x_{1}, x_{2}\right):=\operatorname{dist}\left\{\left(x_{1}, x_{2}\right) \in \bar{\omega}, \partial \omega\right\}
$$

is the distance between $\left(x_{1}, x_{2}\right) \in \bar{\omega}$ and $\partial \omega$, is clear.

Lemma 2.3 The sets (2.6) are Banach spaces, and moreover Hilbert spaces.

Proof. Lemma 2.3 immediately follows from a general theorem of Kufner, Opic, 1984, since

$$
h^{-1}, h^{-3}, x_{2}^{-\kappa}, x_{2}^{-3 \kappa}, x_{2}^{2-\kappa}, d^{-\kappa}, d^{-3 \kappa} \in L_{1}^{\text {loc }}(\omega) \text {. }
$$

Definition 2.4 In the set

$$
W_{2}^{1}\left(\omega, \begin{array}{l}
\rho \\
h
\end{array}\right) \times W_{2}^{1}\left(\omega, \begin{array}{l}
\rho \\
h
\end{array}\right) \times W_{2}^{1}\left(\omega, \begin{array}{l}
h \\
h^{3}
\end{array}\right)
$$


of vector-functions $u:=\left(u_{1}, u_{2}, v_{3}\right)$ such that

$$
u_{\alpha} \in W_{2}^{1}\left(\omega, \begin{array}{l}
\rho \\
h
\end{array}\right), \quad \alpha=1,2 ; \quad v_{3} \in W_{2}^{1}\left(\omega, \begin{array}{c}
h \\
h^{3}
\end{array}\right)
$$

we introduce two norms:

$$
\begin{gathered}
\|u\|_{W_{2, \rho}^{1}}^{2}:=\sum_{\alpha=1}^{2}\left\|u_{\alpha}\right\|_{W_{2}^{1}\left(\omega,,_{h}^{\rho}\right)}^{2}+\left\|v_{3}\right\|_{W_{2}^{1}\left(\omega, h_{h} h^{3}\right.}^{2}, \\
\|u\|_{B W_{2, \rho}^{1}}^{2}:=\int_{\omega}\left\{\rho\left[\left(u_{1}\right)^{2}+\left(u_{2}\right)^{2}\right]+B(u, u)\right\} d \omega,
\end{gathered}
$$

and the corresponding scalar products:

$$
\begin{gathered}
\left(u, u^{*}\right)_{W_{2, \rho}^{1}}:=\sum_{\alpha=1}^{2}\left(u_{\alpha}, u_{\alpha}^{*}\right)_{W_{2}^{1}\left(\omega,{ }_{h}^{\rho}\right)}+\left(v_{3}, v_{3}^{*}\right)_{W_{2}^{1}\left(\omega, h_{h}^{h}\right)}, \\
\left(u, u^{*}\right)_{B} W_{2, \rho}^{1}:=\sum_{\alpha=1}^{2}\left(\rho^{1 / 2} u_{\alpha}, \rho^{1 / 2} u_{\alpha}^{*}\right)_{L_{2}(\omega)}+\int_{\omega} B\left(u, u^{*}\right) d \omega .
\end{gathered}
$$

The obtained spaces will be denoted by

$$
W_{2, \rho}^{1}
$$

and

$$
{ }^{B} W_{2, \rho}^{1}
$$

respectively.

In this paper we consider the spaces (2.14) and (2.15) for

$$
\rho=1, \quad \rho=x_{2}^{\kappa-2}, \quad \rho=h .
$$

Let further

$$
\begin{gathered}
W_{2, \overline{\kappa-2}}^{1}:=W_{2, x_{2}^{\kappa-2},}^{1}, \\
{ }^{B} W_{2, \overline{\kappa-2}}^{1}:={ }^{B} W_{2, x_{2}^{\kappa-2}}^{1} .
\end{gathered}
$$

Evidently, from (2.17), (2.18) for $\kappa=2$ and (2.14), (2.15) for $\rho=1$, we have

$$
\begin{gathered}
W_{2, \overline{0}}^{1}=W_{2,1}^{1}, \\
{ }^{B} W_{2, \overline{0}}^{1}={ }^{B} W_{2,1}^{1} .
\end{gathered}
$$


Theorem 2.5 The spaces (2.19), (2.20),

$$
W_{2, \overline{\kappa-2}}^{1},
$$

and

$$
{ }^{B} W_{2, \overline{\kappa-2}}^{1}
$$

are Hilbert spaces, and we have

$$
\|u\|_{B W_{2, \overline{0}}^{1}\left({ }^{B} W_{2, \overline{\kappa-2}}^{1}\right)}^{2} \leq C\|u\|_{W_{2, \overline{0}}^{1}\left({ }^{B} W_{2, \overline{\kappa-2}}^{1}\right)}^{2}
$$

where

$$
C:=\max \{1,9(3 \lambda+2 \mu)\} .
$$

Proof. The vector spaces (2.14) and (2.21) are Hilbert spaces since so are the spaces

$$
W_{2}^{1}\left(\omega, \begin{array}{l}
h \\
h^{3}
\end{array}\right) \text { and } W_{2}^{1}\left(\omega, \begin{array}{l}
\rho \\
h
\end{array}\right) \text { for } \rho=1 \text { and } \rho=x_{2}^{\kappa-2} .
$$

Let us prove the completeness of the vector spaces (2.15), (2.22) since the scalar products defined by (2.13) and (2.20), obviously, have all the properties of scalar products. Let $\stackrel{n}{u}$ be a fundamental sequence in $W_{2, \overline{0}}^{1}$, i.e.,

$$
\|\stackrel{n}{u}-\stackrel{m}{u}\|_{W_{2, \overline{0}}^{1}} \rightarrow 0 \quad \text { as } \quad n, m \rightarrow+\infty
$$

then from (2.11), taking into account (1.11), we get

$$
\begin{gathered}
\left\|\stackrel{n}{u}_{\alpha}-\stackrel{m}{u}_{\alpha}\right\|_{L_{2}(\omega)} \rightarrow 0, \quad\left\|h\left(\stackrel{n}{u}_{\alpha, \alpha}-\stackrel{m}{u}_{\alpha, \alpha}\right)\right\|_{L_{2}(\omega)} \rightarrow 0, \quad \alpha=1,2, \\
\left\|h\left(\stackrel{n}{v}_{3}-\stackrel{m}{v}_{3}\right)\right\|_{L_{2}(\omega)} \rightarrow 0, \quad\left\|h^{3}\left(\stackrel{n}{v}_{3, \alpha}-\stackrel{m}{v}_{3, \alpha}\right)\right\|_{L_{2}(\omega)} \rightarrow 0, \quad \alpha=1,2, \\
\left\|h\left(u_{2,1}-\stackrel{m}{u}_{2,1}\right)+h\left(\stackrel{n}{u}_{1,2}-\stackrel{m}{u}_{1,2}\right)\right\|_{L_{2}(\omega)} \rightarrow 0, \quad \text { as } \quad n, m \rightarrow+\infty .
\end{gathered}
$$

Replacing $u_{1}$ in (2.11) by $-u_{1}$, we obtain in a similar manner

$$
\left\|h\left(\stackrel{n}{u}_{2,1}-\stackrel{m}{u}_{2,1}\right)-h\left(\stackrel{n}{u}_{1,2}-\stackrel{m}{u}_{1,2}\right)\right\|_{L_{2}(\omega)} \rightarrow 0, \quad \text { as } \quad n, m \rightarrow+\infty .
$$

From (2.27) and (2.28) then follows

$$
\begin{aligned}
\left\|h\left(\stackrel{n}{u}_{2,1}-\stackrel{m}{u}_{2,1}\right)\right\|_{L_{2}(\omega)} & =\frac{1}{2} \| h\left(\stackrel{n}{u}_{2,1}-\stackrel{m}{u}_{2,1}\right)+h\left(\stackrel{n}{u_{1,2}}-\stackrel{m}{u_{1,2}}\right) \\
& +h\left(\stackrel{n}{u}_{2,1}-\stackrel{m}{u}_{2,1}\right)-h\left(\stackrel{n}{u}_{1,2}-\stackrel{m}{u}_{1,2}\right) \|_{L_{2}(\omega)} \\
& \leq \frac{1}{2}\left\|h\left(\stackrel{n}{u}_{2,1}-\stackrel{m}{u}_{2,1}\right)+h\left(\stackrel{n}{u}_{1,2}-\stackrel{m}{u}_{1,2}\right)\right\|_{L_{2}(\omega)} \\
& +\frac{1}{2}\left\|h\left(\stackrel{n}{u}_{2,1}-\stackrel{m}{u}_{2,1}\right)-h\left(\stackrel{n}{u_{1,2}}-\stackrel{m}{u}_{1,2}\right)\right\|_{L_{2}(\omega)} \rightarrow 0
\end{aligned}
$$


as $n, m \rightarrow+\infty$, and, similarly,

$$
\left\|h\left(\stackrel{n}{u}_{1,2}-\stackrel{m}{u}_{1,2}\right)\right\|_{L_{2}(\omega)} \rightarrow 0, \quad \text { as } \quad n, m \rightarrow+\infty
$$

(2.25), (2.29), (2.30), (2.26) mean that $\stackrel{n}{u_{\alpha}}, \alpha=1,2$, and $\stackrel{n}{v_{3}}$ are fundamental sequences in $W_{2}^{1}\left(\omega, \begin{array}{l}1 \\ h\end{array}\right)$ and $W_{2}^{1}\left(\omega, h_{h}^{h}\right)$, respectively. Since the latter spaces are complete there exist elements

$$
u_{\alpha} \in W_{2}^{1}\left(\omega, \begin{array}{l}
1 \\
h
\end{array}\right), \quad \alpha=1,2, \quad v_{3} \in W_{2}^{1}\left(\omega, \begin{array}{c}
h \\
h^{3}
\end{array}\right)
$$

such that

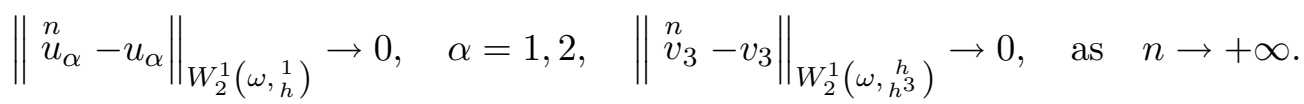

Therefore, on the one hand,

$$
u:=\left(u_{1}, u_{2}, v_{3}\right) \in W_{2, \overline{0}}^{1}
$$

and, on the other hand,

$$
\|\stackrel{n}{u}-u\|_{W_{2, \overline{0}}^{1}} \rightarrow 0, \quad \text { as } \quad n \rightarrow+\infty .
$$

Hence, in view of (2.8), (2.11), (2.15), (2.20),

$$
u \in{ }^{B} W_{2, \overline{0}}^{1}
$$

and, by virtue of

$$
\begin{aligned}
\left\|u^{*}\right\|_{B W_{2, \overline{0}}^{1}}^{2} & \leq \int_{\omega}\left\{\left(u_{1}^{*}\right)^{2}+\left(u_{2}^{*}\right)^{2}+3 \lambda h\left[\left(u_{1,1}^{*}\right)^{2}+\left(u_{2,2}^{*}\right)^{2}+9\left(v_{3}^{*}\right)^{2}\right]\right. \\
& +\mu h\left[2\left(u_{1,1}^{*}\right)^{2}+2\left(u_{2,2}^{*}\right)^{2}+2\left(u_{2,1}^{*}\right)^{2}+2\left(u_{1,2}^{*}\right)^{2}+18\left(v_{3}^{*}\right)^{2}\right] \\
& \left.+3 \mu h^{3}\left[\left(v_{3,1}^{*}\right)^{2}+\left(v_{3,2}^{*}\right)^{2}\right]\right\} d \omega \leq C\left\|u^{*}\right\|_{W_{2, \overline{0}}^{1}}^{2},
\end{aligned}
$$

which is true for any

$$
\begin{gathered}
u^{*}:=\left(u_{1}^{*}, u_{2}^{*}, v_{3}^{*}\right) \in W_{2, \overline{0}}^{1}, \\
\|u-u\|_{B W_{2, \overline{0}}^{1}} \rightarrow 0 \quad \text { as } \quad n \rightarrow+\infty .
\end{gathered}
$$

Thus, along with (2.24) we have (2.32) which means the completeness of the space ${ }^{B} W_{2, \overline{0}}^{1}$. The arguments for the completeness of ${ }^{B} W_{2, \overline{\kappa-2}}^{1}$ are similar. Observe that $(2.23)$ is a consequence of (2.31). 
In what follows we assume

$$
h\left(x_{1}, x_{2}\right) \geq h_{\kappa} x_{2}^{\kappa} \quad \forall\left(x_{1}, x_{2}\right) \in \omega, \quad h_{\kappa}, \kappa=\text { const }>0,
$$

where $\kappa$ denotes the smallest possible exponent. If (2.33) holds for every $\kappa>0$, i.e., a minimal one does not exist, then we assume $\kappa$ as arbitrarily small. An example for such a situation is the case

$$
h\left(x_{1}, x_{2}\right)=h_{0}\left[\ln \left(\frac{l}{x_{2}}\right)\right]^{-1}, \quad h_{0}=\mathrm{const}>0, \quad l>\max _{\left(x_{1}, x_{2}\right) \in \bar{\omega}}\left\{x_{2}\right\} .
$$

In the particular case of (2.33) when

$$
h^{\kappa} x_{2}^{\kappa} \geq h\left(x_{1}, x_{2}\right) \geq h_{\kappa} x_{2}^{\kappa}, \quad h^{\kappa}=\text { const }>0,
$$

it is clear that $\kappa$ is minimal, otherwise we would have a contradiction to the left inequality in (2.35). If $\kappa<\frac{1}{3}$ in (2.33) (for the $\mathrm{N}$-th approximation $\frac{1}{3}$ should be replaced by $\left.\frac{1}{1+2 N}\right)$, it is not necessary to find a minimal $\kappa$, since for any $\kappa<\frac{1}{3}$ we have the same result concerning the traces of $u$ on $\gamma_{0}$ (see below). So, in the case (2.34) we can take any $\kappa<\frac{1}{3}$ in $(2.33)$.

Let

$$
I_{k}\left(x_{1}\right):=\int_{0}^{l\left(x_{1}\right)} h^{-2 k-1} d x_{2} \quad \forall\left(x_{1}, 0\right) \in \gamma_{0}, \quad k=0,1, \ldots,
$$

where

$$
l\left(x_{1}\right):=\max _{\left(x_{1}, x_{2}\right) \in \bar{\omega}}\left\{x_{2}\right\} \quad \text { for a fixed } \quad\left(x_{1}, 0\right) \in \gamma_{0} .
$$

Then:

(i) from $\kappa<\frac{1}{2 k+1}$ it follows that $I_{k}\left(x_{1}\right)<+\infty$ because of

$$
\frac{1}{h^{2 k+1}} \leq \frac{1}{h_{\kappa} x_{2}^{(2 k+1) \kappa}}
$$

in view of $(2.33)$;

(ii) from $I_{k}\left(x_{2}\right)=+\infty$ it follows that $\kappa \geq \frac{1}{2 k+1}$ since otherwise, i.e., if $\kappa<\frac{1}{2 k+1}$, we would have (i) and thus a contradiction.

If $h$ vanishes logarithmically (see, e.g., (2.34)), then (2.33) holds for every $\kappa$. But according to our assumption we can take $0<\kappa<\frac{1}{2 k+1}$.

So, roughly speaking, convergence of $I_{k}\left(x_{1}\right)$ is equivalent to $\kappa<\frac{1}{2 k+1}$ and divergence of $I_{k}\left(x_{1}\right)$ is equivalent to $\kappa \geq \frac{1}{2 k+1}$. 
Lemma 2.6 Under the condition (2.33) we have

$$
\begin{aligned}
& W_{2}^{1}\left(\omega, \begin{array}{c}
1 \\
h
\end{array}\right) \subset W_{2}^{1}\left(\omega, \begin{array}{c}
1 \\
x_{2}^{\kappa}
\end{array}\right) \subset W_{2}^{1}\left(\omega, \begin{array}{c}
1 \\
d^{\kappa}
\end{array}\right), \\
& W_{2}^{1}(\omega, h) \subset W_{2}^{1}\left(\omega, x_{2}^{\kappa}\right) \subset W_{2}^{1}\left(\omega, d^{\kappa}\right), \\
& W_{3}^{1}\left(\omega, \begin{array}{c}
h \\
h^{3}
\end{array}\right) \subset W_{2}^{1}\left(\omega, \begin{array}{c}
x_{2}^{\kappa} \\
x_{2}^{3 \kappa}
\end{array}\right) \subset W_{2}^{1}\left(\omega, x_{2}^{3 \kappa}\right) \subset W_{2}^{1}\left(\omega, d^{3 \kappa}\right), \\
& W_{2}^{1}\left(\begin{array}{c}
1 \\
1 \\
x_{2}^{\kappa}
\end{array}\right) \subset W_{2}^{1}\left(\omega, \begin{array}{c}
1 \\
x_{2}^{2}
\end{array}\right) \quad \text { for } \quad 0<\kappa<2 .
\end{aligned}
$$

Proof. Follows from (2.33) together with the following obvious inequalities:

$$
d\left(x_{1}, x_{2}\right) \leq x_{2} \quad \text { for } \quad\left(x_{1}, x_{2}\right) \in \bar{\omega}
$$

(if $d\left(x_{1}, x_{2}\right)$ is a regularized distance, then in the equality (2.40) there arises a constant factor);

$$
x_{2}^{\kappa_{1}} \leq l^{\kappa_{1}-\kappa_{2}} x_{2}^{\kappa_{2}} \text { for } \quad \kappa_{1}>\kappa_{2}
$$

with $l$ being defined in (2.34). For the proof of (2.39) and the second inclusion of (2.38) we use (2.41) for $\kappa_{1}=2, \kappa_{2}=\kappa$ and $\kappa_{1}=3 \kappa, \kappa_{2}=\kappa$, respectively.

Lemma 2.7 We have the following identities in the sense of equivalent norms

$$
\begin{gathered}
W_{2}^{1}\left(\omega, d^{\kappa}\right)=W_{2}^{1}\left(\omega, \begin{array}{c}
1 \\
d^{\kappa}
\end{array}\right) \quad \text { for } \quad-1<\kappa \leq 2, \\
W_{2}^{1}\left(\omega, d^{3 \kappa}\right)=W_{2}^{1}\left(\omega, \begin{array}{c}
1 \\
d^{3 \kappa}
\end{array}\right) \quad \text { for } \quad-\frac{1}{3}<\kappa \leq \frac{2}{3} .
\end{gathered}
$$

Proof. Immediately follows from Theorem 1.1.4 in Nikolskii et al., 1988.

Lemma 2.8 Let $\partial \omega \in C^{2}$. If

$$
-1<\kappa<1 \quad\left(-\frac{1}{3}<\kappa<\frac{1}{3}\right)
$$

and

$$
\varphi \in W_{2}^{1}\left(\omega, \begin{array}{c}
1 \\
d^{\kappa}
\end{array}\right) \quad\left(\varphi \in W_{2}^{1}\left(\omega, \begin{array}{c}
1 \\
d^{3 \kappa}
\end{array}\right)\right),
$$

then for the trace of $\varphi$ on $\partial \omega$ we have

$$
\left.\varphi\right|_{\partial \omega} \in B_{2}^{\frac{1-\kappa}{2}}(\partial \omega) \quad\left(\left.\varphi\right|_{\partial \omega} \in B_{2}^{\frac{1-3 \kappa}{2}}(\partial \omega)\right),
$$

where B means Besov spaces.

Proof. This lemma is a particular case of Theorem 1.1.2 in Nikolskii et al., 1988. 
Lemma 2.9 Let $\partial \omega \in C^{2}$, assume (2.33), and let

$$
\begin{gathered}
\varphi \in W_{2}^{1}\left(\omega, \begin{array}{l}
1 \\
h
\end{array}\right) \text { or } \varphi \in W_{2}^{1}(\omega, h) \text { for } 0<\kappa<1 \text {, i.e., } I_{0}\left(x_{1}\right)<+\infty \\
\left(\varphi \in W_{2}^{1}\left(\omega, \begin{array}{c}
h \\
h^{3}
\end{array}\right) \text { for } 0<\kappa<\frac{1}{3} \text {, i.e., } I_{1}\left(x_{1}\right)<+\infty\right)
\end{gathered}
$$

Then

$$
\begin{gathered}
\left.\varphi\right|_{\partial \omega} \in B_{2}^{\frac{1-\kappa}{2}}(\partial \omega) \subset L_{2}(\partial \omega) \\
\left(\left.\varphi\right|_{\partial \omega} \in B_{2}^{\frac{1-3 \kappa}{2}}(\partial \omega) \subset L_{2}(\partial \omega)\right) .
\end{gathered}
$$

Proof. Follows from Lemmas $2.6-2.8$.

Note that the existence of the traces on $\partial \omega$ implies the existence of the traces on $\gamma_{0} \in \partial \omega$ and $\gamma \in \partial \omega$ for $\kappa$ as in (2.47) and (2.48) i.e., under the corresponding restrictions on $h$. But as it follows from the following lemma for the existence of the traces on $\gamma$ the above-mentioned restrictions are unnecessary.

Lemma 2.10 Let $\partial \omega \in C^{2}$ and

$$
\varphi \in W_{2}^{1}\left(\omega, \begin{array}{l}
1 \\
h
\end{array}\right) \quad \text { or } \quad \varphi \in W_{2}^{1}(\omega, h) \quad \text { or } \quad \varphi \in W_{2}^{1}\left(\omega, \begin{array}{c}
h \\
h^{3}
\end{array}\right)
$$

Then

$$
\left.\varphi\right|_{\gamma} \in W_{2}^{1 / 2}(\gamma) \subset L_{2}(\gamma)
$$

Proof. Let

$$
\omega_{\delta}:=\left\{\left(x_{1}, x_{2}\right) \in \omega: x_{2}>\delta, \quad \delta=\text { const }>0\right\} .
$$

Evidently,

$$
\begin{aligned}
& \varphi \in W_{2}^{1}\left(\omega, \begin{array}{l}
1 \\
h
\end{array}\right) \subset W_{2}^{1}\left(\omega_{\delta}, \begin{array}{c}
1 \\
h
\end{array}\right)=W_{2}^{1}\left(\omega_{\delta}\right), \\
& \varphi \in W_{2}^{1}(\omega, h) \subset W_{2}^{1}\left(\omega_{\delta}, h\right)=W_{2}^{1}\left(\omega_{\delta}\right), \\
& \varphi \in W_{2}^{1}\left(\omega, \begin{array}{c}
h \\
h^{3}
\end{array}\right) \subset W_{2}^{1}\left(\omega_{\delta}, \begin{array}{c}
h \\
h^{3}
\end{array}\right)=W_{2}^{1}\left(\omega_{\delta}\right),
\end{aligned}
$$

where $W_{2}^{1}\left(\omega_{\delta}\right)$ is the usual Sobolev space and for any $\delta>0$ there exists the trace of $\varphi$ on

$$
\gamma_{\delta}:=\left\{\left(x_{1}, x_{2}\right) \in \gamma: x_{2}>\delta, \quad \delta=\text { const }>0\right\} .
$$


Lemma 2.11 Let $\partial \omega \in C^{2}$. If (2.33) holds and

$$
u \in W_{2, \rho}^{1} \quad \text { for } \rho=1 \text { and } \rho=h,
$$

then $u_{\alpha}, \alpha=1,2$, belong to the spaces (2.36), or (2.37), respectively, $v_{3}$ belongs to the space (2.38) and consequently to the space (2.43); for the the traces we have

$$
\begin{gathered}
\left.u\right|_{\gamma} \in\left[W_{2}^{1 / 2}(\gamma)\right]^{3} \subset\left[L_{2}(\gamma)\right]^{3}, \\
\left.u_{\alpha}\right|_{\gamma_{0}} \in B_{2}^{\frac{1-\kappa}{2}}\left(\gamma_{0}\right) \subset L_{2}\left(\gamma_{0}\right), \quad \alpha=1,2, \text { for } 0<\kappa<1 \text {, i.e., } I_{0}\left(x_{1}\right)<+\infty, \\
\left.v_{3}\right|_{\gamma_{0}} \in B_{2}^{\frac{1-3 \kappa}{2}}\left(\gamma_{0}\right) \subset L_{2}\left(\gamma_{0}\right) \text { for } 0<\kappa<\frac{1}{3} \text {, i.e., } I_{1}\left(x_{1}\right)<+\infty
\end{gathered}
$$

Proof. Follows from Lemmas 2.6, 2.7, 2.9, 2.10.

Finally, let us introduce the space

$$
\stackrel{\circ}{W_{2}}\left(\omega, \begin{array}{c}
x_{2}^{\kappa-2} \\
x_{2}^{\kappa}
\end{array}\right)
$$

as the closure of $C_{0}^{\infty}(\omega)$ with respect to the norm of

$$
W_{2}^{1}\left(\omega, \begin{array}{c}
x_{2}^{\kappa-2} \\
x_{2}^{\kappa}
\end{array}\right)
$$

\section{Lemma 2.12 If}

$$
\varphi \in \stackrel{\circ}{W_{2}}\left(\omega, \begin{array}{c}
x_{2}^{\kappa-2} \\
x_{2}^{\kappa}
\end{array}\right)
$$

then for the trace we have

$$
\left.\varphi\right|_{\gamma}=0 .
$$

Proof. From (2.58) we get

$$
\left\|x_{2}^{\frac{\kappa-2}{2}} \varphi\right\|_{L_{2}(\omega)}<+\infty, \quad\left\|x_{2}^{\frac{\kappa}{2}} \varphi_{, \alpha}\right\|_{L_{2}(\omega)}<+\infty, \quad \alpha=1,2,
$$

and there exist $\varphi_{n} \in C_{0}^{\infty}(\omega)$ such that

$$
\left\|x_{2}^{\frac{\kappa-2}{2}}\left(\varphi_{n}-\varphi\right)\right\|_{L_{2}(\omega)} \rightarrow 0, \quad\left\|x_{2}^{\frac{\kappa}{2}}\left(\varphi_{n, \alpha}-\varphi_{, \alpha}\right)\right\|_{L_{2}(\omega)} \rightarrow 0 \alpha=1,2, \text { as } n \rightarrow \infty
$$

with

$$
\left.\varphi_{n}\right|_{\gamma}=0,\left.\quad \varphi_{n}\right|_{\gamma_{0}}=0 .
$$

Consider the spaces defined by the following restrictions:

$$
\widetilde{\varphi}:=\left.\varphi\right|_{\omega_{\delta}}, \quad \widetilde{\varphi}_{n}:=\left.\varphi_{n}\right|_{\omega_{\delta}} .
$$


Then, by virtue of (2.59), (2.60),

$$
\begin{gathered}
\left\|x_{2}^{\frac{\kappa-2}{2}} \widetilde{\varphi}\right\|_{L_{2}\left(\omega_{\delta}\right)}<+\infty, \quad\left\|x_{2}^{\frac{\kappa}{2}} \widetilde{\varphi}_{, \alpha}\right\|_{L_{2}\left(\omega_{\delta}\right)}<+\infty, \quad \alpha=1,2, \\
0 \leq\left\|x_{2}^{\frac{\kappa-2}{2}}\left(\widetilde{\varphi}_{n}-\widetilde{\varphi}\right)\right\|_{L_{2}\left(\omega_{\delta}\right)} \leq\left\|x_{2}^{\frac{\kappa-2}{2}}\left(\varphi_{n}-\varphi\right)\right\|_{L_{2}(\omega)} \rightarrow 0, \text { as } n \rightarrow \infty, \quad \text {, } \\
0 \leq\left\|x_{2}^{\frac{\kappa}{2}}\left(\widetilde{\varphi}_{n, \alpha}-\widetilde{\varphi}_{, \alpha}\right)\right\|_{L_{2}\left(\omega_{\delta}\right)} \leq\left\|x_{2}^{\frac{\kappa}{2}}\left(\varphi_{n, \alpha}-\varphi_{, \alpha}\right)\right\|_{L_{2}(\omega)} \rightarrow 0, \alpha=1,2, \quad \text { as } n \rightarrow \infty .
\end{gathered}
$$

This implies

$$
\left\|x_{2}^{\frac{\kappa-2}{2}}\left(\widetilde{\varphi}_{n}-\widetilde{\varphi}\right)\right\|_{L_{2}\left(\omega_{\delta}\right)} \rightarrow 0,\left\|x_{2}^{\frac{\kappa}{2}}\left(\widetilde{\varphi}_{n, \alpha}-\widetilde{\varphi}_{, \alpha}\right)\right\|_{L_{2}\left(\omega_{\delta}\right)} \rightarrow 0, \alpha=1,2, \quad \text { as } n \rightarrow \infty
$$

and, because of the boundedness of $x_{2}^{\frac{\kappa-2}{2}}, x_{2}^{\frac{\kappa}{2}}$ on $\omega_{\delta}$,

$$
\left\|\widetilde{\varphi}_{n}-\widetilde{\varphi}\right\|_{L_{2}\left(\omega_{\delta}\right)} \rightarrow 0, \quad\left\|\widetilde{\varphi}_{n, \alpha}-\widetilde{\varphi}_{, \alpha}\right\|_{L_{2}\left(\omega_{\delta}\right)} \rightarrow 0, \quad \alpha=1,2, \quad \text { as } n \rightarrow \infty .
$$

So, the set of restrictions $\widetilde{\varphi}_{n} \in W_{2}^{1}\left(\omega_{\delta}\right)$ and, therefore, $\widetilde{\varphi}$ has a trace on $\partial \omega_{\delta}$, which, in view of the first property from (2.61), is equal to 0 on $\gamma_{\delta}$, i.e.,

$$
\left.\widetilde{\varphi}\right|_{\gamma_{\delta}}=0, \quad \text { i.e., }\left.\quad \varphi\right|_{\gamma_{\delta}}=0, \quad \text { for any } \delta>0 .
$$

Since $\delta$ is arbitrary, from (2.65) it follows that

$$
\left.\varphi\right|_{\gamma}=0 \text {. }
$$

The trace of $\varphi$ on $\gamma_{0}$ does not exist, in general.

Lemma 2.13 Let $\kappa>1$. The space

$$
\stackrel{\circ}{W_{2}}\left(\omega, \begin{array}{c}
x_{2}^{\kappa-2} \\
x_{2}^{\kappa}
\end{array}\right)
$$

coincides with

$$
\left\{\varphi \in W_{2}^{1}\left(\omega, \begin{array}{c}
x_{2}^{\kappa-2} \\
x_{2}^{\kappa}
\end{array}\right):\left.\varphi\right|_{\gamma}=0\right\}
$$

Note that both the spaces (2.67) and (2.68) consist of functions with the properties (2.59), (2.66), and the same norm; both are complete.

The proof of Lemma 2.13 is analogous to the proof of the well-known results

$$
W_{2}^{1}\left(\omega, \begin{array}{c}
d^{\kappa-2} \\
d^{\kappa}
\end{array}\right)=W_{2}^{1}\left(\omega, \begin{array}{c}
1 \\
d^{\kappa}
\end{array}\right)=\stackrel{\stackrel{1}{W}_{2}}{ }\left(\omega, \begin{array}{c}
1 \\
d^{\kappa}
\end{array}\right) \text { for } \quad|\kappa|>1 .
$$

The traces on $\partial \omega$ do not exist, in general (see Nikolskii et.al 1988, Theorem 1.2.4 and references therein). Note as well that

$$
\left\{\varphi \in \stackrel{\circ}{W}_{2}^{1}\left(\omega, \begin{array}{c}
1 \\
d^{\kappa}
\end{array}\right)\right\}=\left\{\varphi \in W_{2}^{1}\left(\omega, \begin{array}{c}
1 \\
d^{\kappa}
\end{array}\right):\left.\varphi\right|_{\partial \omega}=0\right\} \quad \text { for } \quad|\kappa|<1
$$

(see ibid, Theorem 1.2.1 and compare with Lemmas 2.12, 2.13 of the present paper). 
Lemma 2.14 Under the condition (2.33) we have

$$
W_{2}^{1}\left(\omega, \begin{array}{c}
x_{2}^{\kappa-2} \\
h
\end{array}\right) \subset W_{2}^{1}\left(\omega, \begin{array}{c}
x_{2}^{\kappa-2} \\
x_{2}^{\kappa}
\end{array}\right)
$$

Proof. Is evident.

\section{$3 \quad$ Hardy's Inequality}

\section{Lemma 3.1 If}

$$
\varphi \in W_{2}^{1}\left(\omega, \begin{array}{c}
x_{2}^{\kappa-2} \\
x_{2}^{\kappa}
\end{array}\right)
$$

and

$$
\left.\varphi\right|_{\gamma}=0
$$

then

$$
\int_{\omega} x_{2}^{\kappa-2} \varphi^{2}\left(x_{1}, x_{2}\right) d \omega \leq \frac{4}{(\kappa-1)^{2}} \int_{\omega} x_{2}^{\kappa}\left[\varphi_{, 2}\left(x_{1}, x_{2}\right)\right]^{2} d \omega \quad \text { for } \quad \kappa>1 .
$$

Proof. In what follows, without loss of generality, we suppose that the domain $\omega$ lies inside the rectangle

$$
\Pi:=\left\{\left(x_{1}, x_{2}\right) \in R^{2}: a<x_{1}<b, 0<x_{2}<l\right\} .
$$

Let us complete a definition of the function $\varphi$ in $\Pi \backslash \Omega$, assuming $\varphi$ to be equal to zero here. Then (3.1) obviously implies

$$
\int_{\Pi}\left[x_{2}^{\kappa-2} \varphi^{2}+x_{2}^{\kappa}(\varphi, 2)^{2}\right] d \Pi<+\infty,
$$

i.e., according to Fubini's theorem, for almost every $\left.x_{1} \in\right] a, b[$

$$
\varphi\left(x_{1}, \cdot\right) \in W_{2}^{1}(] 0, l\left[, x_{2}^{\kappa-2}, x_{2}^{\kappa}\right),
$$

which is a weighted Sobolev space with the norm

$$
\left\|\varphi\left(x_{1,} \cdot\right)\right\|_{W_{2}^{1}(] 0, l\left[x_{2}^{\kappa-2}, x_{2}^{\kappa}\right)}^{2}=\int_{0}^{l}\left\{x_{2}^{\kappa-2} \varphi^{2}\left(x_{1}, \cdot\right)+x_{2}^{\kappa}\left[\varphi_{, 2}\left(x_{1}, \cdot\right)\right]^{2}\right\} d x_{2} .
$$

Evidently,

$$
W_{2}^{1}(] \delta, l\left[, x_{2}^{\kappa-2}, x_{2}^{\kappa}\right)=W_{2}^{1}(] \delta, l[) .
$$

Hence, since in view of (3.2), for almost every $\left.x_{1} \in\right] a, b[$

$$
\varphi\left(x_{1}, l\right)=0,
$$


we have

$$
\varphi\left(x_{1}, .\right) \in A C_{R}(\delta, l)
$$

(i.e., for almost every $\left.x_{1} \in\right] a, b[$ the function $\varphi$ is absolutely continuous with respect to $x_{2} \in[\delta, l]$ and satifies (3.6)). Therefore (see Opic, Kufner, 1992), for almost every $\left.x_{1} \in\right] a, b[$,

$$
\int_{\delta}^{l} x_{2}^{\kappa-2}\left[\varphi\left(x_{1}, x_{2}\right)\right]^{2} d x_{2} \leq \frac{4}{(\kappa-1)^{2}} \int_{\delta}^{l} x_{2}^{\kappa}\left[\varphi_{, 2}\left(x_{1}, x_{2}\right)\right]^{2} d x_{2} \quad \text { for } \quad \kappa>1 .
$$

Now, considering the limit procedure as $\delta \rightarrow 0_{+}$, since the limits of the integrals in (3.8) exist for almost every $\left.x_{1} \in\right] a, b[$ because of (3.5), we get

$$
\int_{0}^{l} x_{2}^{\kappa-2}\left[\varphi\left(x_{1}, x_{2}\right)\right]^{2} d x_{2} \leq \frac{4}{(\kappa-1)^{2}} \int_{0}^{l} x_{2}^{\kappa}\left[\varphi_{, 2}\left(x_{1}, x_{2}\right)\right]^{2} d x_{2} \quad \text { for } \quad \kappa>1
$$

for almost every fixed $\left.x_{1} \in\right] a, b\left[\right.$. Integrating both the sides of (3.9) by $x_{1}$ over $] a, b[$, we obtain

$$
\begin{aligned}
\int_{\omega} x_{2}^{\kappa-2} \varphi^{2} d \omega & =\int_{\Pi} x_{2}^{\kappa-2} \varphi^{2} d x_{1} d x_{2} \\
& \leq \frac{4}{(\kappa-1)^{2}} \int_{\Pi} x_{2}^{\kappa}\left(\varphi_{, 2}\right)^{2} d x_{1} d x_{2} \\
& =\frac{4}{(\kappa-1)^{2}} \int_{\omega} x_{2}^{\kappa}\left(\varphi_{, 2}\right)^{2} d \omega \text { for } \kappa>1 .
\end{aligned}
$$

\section{Corollary 3.2 If}

$$
\varphi \in W_{2}^{1}\left(\omega, \begin{array}{c}
1 \\
x_{2}^{2}
\end{array}\right)
$$

and (3.2) is fulfilled, then

$$
\int_{\omega} \varphi^{2} d \omega \leq 4 \int_{\omega} x_{2}^{2}(\varphi, 2)^{2} d \omega
$$

Proof. Using the Lemma 3.1 for $\kappa=2$, we get the Corollary 3.2.

Remark 3.3 Since

$$
W_{2}^{1}\left(\omega_{\delta}, \begin{array}{c}
1 \\
x_{2}^{\kappa}
\end{array}\right)=W_{2}^{1}\left(\omega_{\delta}\right),
$$


for

with (3.2) from (3.8) we get

$$
\varphi \in W_{2}^{1}\left(\omega_{\delta}, \begin{array}{c}
1 \\
x_{2}^{\kappa}
\end{array}\right)
$$

$$
\int_{\omega_{\delta}} x_{2}^{\kappa-2} \varphi^{2} d \omega \leq \frac{4}{(\kappa-1)^{2}} \int_{\omega_{\delta}} x_{2}^{\kappa}\left(\varphi_{, 2}\right)^{2} d \omega \quad \text { for } \quad \kappa>1 .
$$

As from (3.11) there follows (3.7), the assertion becomes obvious.

\section{Korn's Weighted Inequality}

Lemma 4.1 (Korn's weighted inequality). Let $\kappa>1$, and

$$
u_{\alpha} \in \stackrel{\circ}{W}_{2}^{1}\left(\omega, \begin{array}{c}
x_{2}^{\kappa-2} \\
x_{2}^{\kappa}
\end{array}\right), \quad \alpha=1,2
$$

then

$$
\begin{aligned}
& \int_{\omega} x_{2}^{\kappa}\left[\left(u_{1,1}\right)^{2}+\left(u_{2,2}\right)^{2}+\left(u_{1,2}\right)^{2}+\left(u_{2,1}\right)^{2}\right] d \omega \\
& \leq C \int_{\omega} x_{2}^{\kappa}\left[\left(u_{1,1}\right)^{2}+\left(u_{2,2}\right)^{2}+\left(u_{1,2}+u_{2,1}\right)^{2}\right] d \omega,
\end{aligned}
$$

where

$$
C:=\max \left\{2+\kappa, 2+\frac{4 \kappa}{(\kappa-1)^{2}}\right\} .
$$

Proof. By virtue of the definition of the space (2.57), there exist

$$
\stackrel{n}{u_{\alpha}} \in C_{0}^{\infty}(\omega)
$$

such that

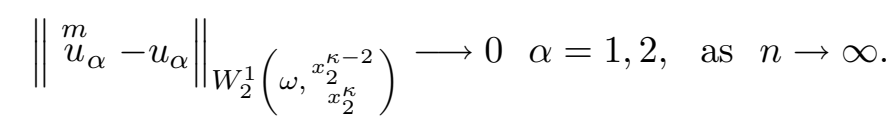

After integration by parts, taking into account (4.4), we get

$$
\begin{aligned}
\int_{\omega} x_{2}^{\kappa} \stackrel{m}{u_{1,2}} \stackrel{m}{u_{2,1}} d \omega & =-\int_{\omega} x_{2}^{\kappa} \stackrel{m}{u_{1,12}} \stackrel{m}{u_{2}} d \omega \\
& =\kappa \int_{\omega} x_{2}^{\kappa-1} \stackrel{m}{u_{1,1}} \stackrel{m}{u}_{2} d \omega+\int_{\omega} x_{2}^{\kappa} \stackrel{m}{u_{1,1}} \stackrel{m}{u_{2,2}} d \omega .
\end{aligned}
$$

On the one hand,

$$
\left|\int_{\omega} x_{2}^{\kappa} \stackrel{m}{u_{1,1}} \stackrel{m}{u_{2,2}} d \omega\right| \leq \frac{1}{2} \int_{\omega} x_{2}^{\kappa}\left[\left(\stackrel{m}{u}_{1,1}\right)^{2}+\left(\stackrel{m}{u}_{2,2}\right)^{2}\right] d \omega .
$$


On the other hand, according to Lemma 2.13, we can use Hardy's inequality (see Lemma 3.1):

$$
\begin{aligned}
\left|\int_{\omega} x_{2}^{\kappa-1} \stackrel{m}{u_{1,1}} \underset{u_{2}}{m} d \omega\right| & =\left|\int_{\omega} x_{2}^{\frac{\kappa}{2}} \stackrel{m}{u}_{1,1} x_{2}^{\frac{\kappa-2}{2}} \stackrel{m}{u}_{2} d \omega\right| \\
& \leq \frac{1}{2}\left[\int_{\omega} x_{2}^{\kappa}\left(\stackrel{m}{u}_{1,1}\right)^{2} d \omega+\int_{\omega} x_{2}^{\kappa-2}\left(\stackrel{m}{u}_{2}\right)^{2} d \omega\right] \\
& \leq \frac{1}{2}\left[\int_{\omega} x_{2}^{\kappa}\left(\stackrel{m}{u}_{1,1}\right)^{2} d \omega+\frac{4}{(\kappa-1)^{2}} \int_{\omega} x_{2}^{\kappa}\left(\stackrel{m}{u}_{2,2}\right)^{2} d \omega\right] .
\end{aligned}
$$

Substituing the estimates $(4.7),(4.8)$ in $(4.6)$, we obtain

$$
\begin{aligned}
& \left|\int_{\omega} x_{2}^{\kappa}{\stackrel{m}{u_{1,2}}}_{u_{2,1}}^{m} d \omega\right| \\
& \quad \leq \frac{1+\kappa}{2} \int_{\omega} x_{2}^{\kappa}\left(\stackrel{m}{u}_{1,1}\right)^{2} d \omega+\frac{1}{2}\left[1+\frac{4 \kappa}{(\kappa-1)^{2}}\right] \int_{\omega} x_{2}^{\kappa}\left(\stackrel{m}{u}_{2,2}\right)^{2} d \omega \\
& \quad \leq \frac{1}{2} C_{1} \int_{\omega} x_{2}^{\kappa}\left[\left(\stackrel{m}{u}_{1,1}\right)^{2}+\left(\stackrel{m}{u}_{2,2}\right)^{2}\right] d \omega,
\end{aligned}
$$

where

$$
C_{1}:=\max \left\{1+\kappa, 1+\frac{4 \kappa}{(\kappa-1)^{2}}\right\} .
$$

For an arbitrary $\varepsilon \in] 0,1[$, in view of (4.9), we have

$$
\begin{aligned}
& \int_{\omega} x_{2}^{\kappa}\left[\left(\stackrel{m}{u}_{1,1}\right)^{2}+\left(\stackrel{m}{u}_{2,2}\right)^{2}+\left(\stackrel{m}{u}_{1,2}+\stackrel{m}{u}_{2,1}\right)^{2}\right] d \omega \\
& \geq \int_{\omega} x_{2}^{\kappa}\left[\left(\stackrel{m}{u}_{1,1}\right)^{2}+\left(\stackrel{m}{u}_{2,2}\right)^{2}+\varepsilon\left(\stackrel{m}{u}_{1,2}+\stackrel{m}{u}_{2,1}\right)^{2}\right] d \omega \\
& =\int_{\omega} x_{2}^{\kappa}\left[\left(\stackrel{m}{u}_{1,1}\right)^{2}+\left(\stackrel{m}{u}_{2,2}\right)^{2}+\varepsilon\left(\stackrel{m}{u}_{1,2}\right)^{2}+\varepsilon\left(\stackrel{m}{u}_{2,1}\right)^{2}+2 \varepsilon \stackrel{m}{u_{1,2}} \stackrel{m}{u_{2,1}}\right] d \omega \\
& \geq \int_{\omega} x_{2}^{\kappa}\left[\left(\stackrel{m}{u}_{1,1}\right)^{2}+\left(\stackrel{m}{u_{2,2}}\right)^{2}+\varepsilon\left(\stackrel{m}{u_{1,2}}\right)^{2}+\varepsilon\left(\stackrel{m}{u_{2,1}}\right)^{2}-2 \varepsilon\left|\int_{\omega} x_{2}^{\kappa} \stackrel{m}{u_{1,2}} \underset{m}{u_{2,1}} d \omega\right|\right. \\
& \geq \int_{\omega} x_{2}^{\kappa}\left[\left(\stackrel{m}{u}_{1,1}\right)^{2}+\left(\stackrel{m}{u}_{2,2}\right)^{2}+\varepsilon\left(\stackrel{m}{u}_{1,2}\right)^{2}+\varepsilon\left(\stackrel{m}{u}_{2,1}\right)^{2}\right] d \omega \\
& -\varepsilon C_{1} \int_{\omega} x_{2}^{\kappa}\left[\left(\stackrel{m}{u}_{1,1}\right)^{2}+\left(\stackrel{m}{u_{2,2}}\right)^{2}\right] d \omega \\
& =\int_{\omega} x_{2}^{\kappa}\left\{\left(1-\varepsilon C_{1}\right)\left[\left(\stackrel{m}{u}_{1,1}\right)^{2}+\left(\stackrel{m}{u}_{2,2}\right)^{2}\right]+\varepsilon\left[\left(\stackrel{m}{u}_{1,2}\right)^{2}+\left(\stackrel{m}{u}_{2,1}\right)^{2}\right]\right\} d \omega \\
& =\frac{1}{1+C_{1}} \int_{\omega} x_{2}^{\kappa}\left[\left(\stackrel{m}{u}_{1,1}\right)^{2}+\left(\stackrel{m}{u}_{2,2}\right)^{2}+\left(\stackrel{m}{u_{1,2}}\right)^{2}+\left(\stackrel{m}{u_{2,1}}\right)^{2}\right] d \omega
\end{aligned}
$$


if we put

$$
\varepsilon=\frac{1}{1+C_{1}}
$$

in the penultimate integral.

Now, taking $m \rightarrow \infty$ on both the sides of (4.11) under the norm of $L_{2}(\omega)$, we get (4.2) because of $x_{2}^{\frac{\kappa}{2}} u_{\alpha, \beta} \in L_{2}(\omega)$ and $x_{2}^{\frac{\kappa}{2}}\left(u_{1,2}+u_{2,1}\right) \in L_{2}(\omega)$.

Remark 4.2 In the particular case $\kappa=2$, i.e.,

$$
u_{\alpha} \in \stackrel{\circ}{W}_{2}^{1}\left(\omega, \begin{array}{c}
1 \\
x_{2}^{2}
\end{array}\right)
$$

from (4.2), (4.3) we have

$$
\begin{aligned}
& \int_{\omega} x_{2}^{2}\left[\left(u_{1,1}\right)^{2}+\left(u_{2,2}\right)^{2}+\left(u_{1,2}\right)^{2}+\left(u_{2,1}\right)^{2}\right] d \omega \\
& \leq 10 \int_{\omega} x_{2}^{2}\left[\left(u_{1,1}\right)^{2}+\left(u_{2,2}\right)^{2}+\left(u_{1,2}+u_{2,1}\right)^{2}\right] d \omega .
\end{aligned}
$$

\section{$5 \quad$ Weighted Boundary Value Problems}

We study cos and sin vibrations

$$
\begin{gathered}
u_{\alpha}\left(x_{1}, x_{2}, t\right)=u_{\alpha}\left(x_{1}, x_{2}\right)\left\{\begin{array}{l}
\cos c t \\
\sin c t
\end{array} \quad, \quad \alpha=1,2,\right. \\
v_{3}\left(x_{1}, x_{2}, t\right)=v_{3}\left(x_{1}, x_{2}\right)\left\{\begin{array}{l}
\cos c t \\
\sin c t
\end{array}\right.
\end{gathered}
$$

The 3D expressions for the displacement vector components $U_{i}\left(x_{1}, x_{2}, x_{3}, t\right), i=$ $1,2,3$, corresponding to the $N=1$ approximation have the following form

$$
\begin{gathered}
U_{\alpha}\left(x_{1}, x_{2}, x_{3}, t\right)=\frac{1}{2} u_{\alpha}\left(x_{1}, x_{2}\right)\left\{\begin{array}{l}
\cos c t \\
\sin c t
\end{array},\right. \\
U_{3}\left(x_{1}, x_{2}, x_{3}, t\right)=\frac{3}{2} x_{3} v_{3}\left(x_{1}, x_{2}\right)\left\{\begin{array}{l}
\cos c t \\
\sin c t
\end{array}\right.
\end{gathered}
$$

The initial conditions, e.g., for $t=0$, look like

$$
\begin{gathered}
u_{\alpha}\left(x_{1}, x_{2}, 0\right)=u_{\alpha}\left(x_{1}, x_{2}\right), \quad v_{3}\left(x_{1}, x_{2}, 0\right)=v_{3}\left(x_{1}, x_{2}\right), \\
\frac{\partial u_{\alpha}\left(x_{1}, x_{2}, 0\right)}{\partial t}=0, \quad \frac{\partial v_{3}\left(x_{1}, x_{2}, 0\right)}{\partial t}=0
\end{gathered}
$$

and

$$
u_{\alpha}\left(x_{1}, x_{2}, 0\right)=0, \quad v_{3}\left(x_{1}, x_{2}, 0\right)=0,
$$




$$
\frac{\partial u_{\alpha}\left(x_{1}, x_{2}, 0\right)}{\partial t}=u_{\alpha}\left(x_{1}, x_{2}\right), \quad \frac{\partial v_{3}\left(x_{1}, x_{2}, 0\right)}{\partial t}=v_{3}\left(x_{1}, x_{2}\right),
$$

for cos and sin vibrations, respectively, and they are automatically fulfilled, provided that BVPs for $u:=\left(u_{1}, u_{2}, v_{3}\right)$ are solved. Hence, we have to consider only BVPs for $u$.

Problem 5.1 Let us consider for the system (1.1) the following BCs:

- on $\gamma$

$$
u=g
$$

- on $\gamma_{0}$ either

$$
\begin{gathered}
u_{\alpha}=u_{\alpha}^{0}, \quad \alpha=1,2 \text { iff } I_{0}\left(x_{1}\right)<+\infty(\kappa<1), \\
v_{3}=v_{3}^{0}, \quad \text { iff } I_{1}\left(x_{1}\right)<+\infty\left(\kappa<\frac{1}{3}\right),
\end{gathered}
$$

or

$$
\begin{gathered}
X_{n \alpha 0}=0, \quad \alpha=1,2, \\
h X_{n 31}=0,
\end{gathered}
$$

or

$$
(5.2), \quad(5.5)
$$

or

$$
(5.4), \quad(5.3)
$$

or

$$
\text { (5.2) for } \quad \alpha=1, \quad(5.4) \text { for } \quad \alpha=2, \quad(5.5)
$$

or

$$
\text { (5.4) for } \alpha=1, \quad(5.2) \quad \text { for } \quad \alpha=2 \text {, }
$$

or

$$
\text { (5.2) for } \alpha=1, \quad(5.4) \text { for } \quad \alpha=2 \text {, }
$$

or

$$
\text { (5.4) for } \alpha=1, \quad(5.2) \text { for } \quad \alpha=2 \text {, }
$$

or on different parts of $\gamma_{0}$ different $B C s$

$$
\text { (5.2), (5.3); (5.4), (5.5); (5.6); (5.7); (5.8); (5.9); (5.10); (5.11) }
$$

are given, where $g$ and $u^{0}:=\left(u_{1}^{0}, u_{2}^{0}, v_{3}^{0}\right)$ are the traces of a prescribed vector

$$
u^{0} \in \begin{cases}{ }^{B} W_{2, \overline{0}}^{1} & \text { for } \quad 0<\kappa \leq 2, \\ { }^{B} W_{2, \overline{\kappa-2}}^{1} & \text { for } \quad \kappa>2\end{cases}
$$

on $\gamma$ and $\gamma_{0}$, respectively.

Since $h \geq 0$, in view of (1.7) - (1.9), BCs (5.4), (5.5) are weighted Neumann type $B C s$ and they shoud be understood as limits from $\omega$ to $\gamma_{0}$. 


$$
\begin{aligned}
& \text { Let } \\
& V_{h}^{0}:=\left\{u^{*}:=\left(u_{1}^{*}, u_{2}^{*}, v_{3}^{*}\right) \in{ }^{B} W_{2, \overline{0}}^{1}:\left.u^{*}\right|_{\gamma}=0\right. \text { and } \\
& \left.u_{\alpha}^{*}\right|_{\gamma_{0}}=0, \quad \alpha=1,2 \text {, when } I_{0}\left(x_{1}\right)<+\infty(\kappa<1) \\
& \text { provided that } u_{\alpha}, \alpha=1,2 \text {, are prescribed on } \gamma_{0} \text { in BCs } \\
& \left.v_{3}^{*}\right|_{\gamma_{0}}=0 \text {, when } I_{1}\left(x_{1}\right)<+\infty\left(\kappa<\frac{1}{3}\right) \\
& \text { provided that } \left.v_{3} \text { is presribed on } \gamma_{0} \text { in BCs in the sense of traces }\right\}
\end{aligned}
$$

and

$$
V_{h}^{\kappa-2}:=\left\{u^{*} \in{ }^{B} W_{2, \overline{\kappa-2}}^{1}:\left.u^{*}\right|_{\gamma}=0 \text { in the sense of traces }\right\}
$$

Obviously, $V_{h}^{0}$ and $V_{h}^{\kappa-2}$ are Hilbert spaces.

Definition 5.2 Let

$$
f_{\alpha} \in L_{2}(\omega), \quad \alpha=1,2, \quad h^{-\frac{1}{2}} f_{3} \in L_{2}(\omega),
$$

and

$$
\kappa<2, \quad \text { i.e., } \quad \int_{0}^{l\left(x_{1}\right)} \tau h^{-1}\left(x_{1}, \tau\right) d \tau<+\infty \text {. }
$$

A function

$$
u \in{ }^{B} W_{2, \overline{0}}^{1}
$$

will be called a weak solution of the Problem 5.1 (actually, there are several BVPs indicated there) if

$$
u-u^{0} \in V_{h}^{0}
$$

and (see (1.6) and (1.20))

$$
J_{c}\left(u, u^{*}\right):=\int_{\omega} B_{c}\left(u, u^{*}\right) d \omega=-\int_{\omega} f u^{*} d \omega \quad \forall u^{*} \in V_{h}^{0} .
$$

Definition 5.3 Let

$$
x_{2}^{\frac{2-\kappa}{2}} f_{\alpha} \in L_{2}(\omega), \quad \alpha=1,2, \quad h^{-\frac{1}{2}} f_{3} \in L_{2}(\omega),
$$

and

$$
\int_{0}^{l\left(x_{1}\right)} \tau h^{-1}\left(x_{1}, \tau\right) d \tau=+\infty . \quad \text { i.e., } \quad \kappa \geq 2 .
$$

A function

$$
u \in{ }^{B} W_{2, \overline{\kappa-2}}^{1}
$$


will be called a weak solution of the Problem 5.1 if

$$
u-u^{0} \in V_{h}^{\kappa-2}
$$

and

$$
J_{c}\left(u, u^{*}\right):=-\int_{\omega} f u^{*} d \omega \quad \forall u^{*} \in V_{h}^{\kappa-2} .
$$

\section{Existence and Uniqueness Theorems}

Theorem 6.1 If (5.17), (5.16) are fulfilled,

$$
p h^{\alpha} \in C(\bar{\omega}), \quad \alpha=1,2,
$$

and

$$
c^{2}<\frac{6 \mu h_{\kappa}}{240 l^{2-\kappa} \max _{\bar{\omega}} p h+h_{\kappa} \max _{\bar{\omega}} p h^{2}},
$$

then there exists a unique weak solution of the Problem 5.1 (more precisely, of each $B V P$ mentioned there) such that

$$
\|u\|_{B} W_{2, \overline{0}}^{1} \leq C_{1}\left[\sum_{\alpha=1}^{2}\left\|f_{\alpha}\right\|_{L_{2}(\omega)}+\left\|h^{-\frac{1}{2}} f_{3}\right\|_{L_{2}(\omega)}+\left\|u^{0}\right\|_{B} W_{2, \overline{0}}^{1}\right],
$$

where the constant $C_{1}$ is independent of $f$ and $u^{0}$.

Theorem 6.2 If (5.22), (5.21) are fulfilled,

$$
p h^{\alpha} x_{2}^{2-\kappa} \in C(\bar{\omega}), \quad \alpha=1,2
$$

and

$$
c^{2}<\frac{6 \mu h_{\kappa}(\kappa-1)^{2}}{24 C \max _{\bar{\omega}}\left(x_{2}^{2-\kappa} p h\right)+h_{\kappa}(\kappa-1)^{2} \max _{\bar{\omega}}\left(p h^{2}\right)},
$$

then there exists a unique weak solution of the Problem 5.1 (more precisely, of one BVP mentioned there, when on $\gamma_{0}$ homogeneous BCs (5.4), (5.5) are set; the other $B V P s$ for (5.22) are not admissible) such that

$$
\|u\|_{B W_{2, \bar{\kappa}-2}} \leq C_{2}\left[\sum_{\alpha=1}^{2}\left\|x_{2}^{\frac{2-\kappa}{2}} f_{\alpha}\right\|_{L_{2}(\omega)}+\left\|h^{-\frac{1}{2}} f_{3}\right\|_{L_{2}(\omega)}+\left\|u^{0}\right\|_{B W_{2, \overline{\kappa-2}}^{1}}\right],
$$

where the constant $C_{2}$ is independent of $f$ and $u^{0}$.

Remark 6.3 In the static case, i.e., when $c=0$, conditions (6.2) and (6.5) are evidently fulfilled. Therefore, the existence and uniqueness theorems for the static case follow from the Theorems 6.1 and 6.2 . 
Proof of Theorem 6.1: Let $u^{*}:=\left(u_{1}^{*}, u_{2}^{*}, v_{3}^{*}\right)$ and

$$
u^{*} \in V_{h}^{0} \text {, }
$$

then

$$
u_{\alpha}^{*} \in W_{2}^{1}\left(\omega, \frac{1}{h}\right), \quad \alpha=1,2,
$$

and

$$
\left.u_{\alpha}^{*}\right|_{\gamma}=0
$$

By virtue of (2.36), (2.39), from (6.8) it follows that

$$
u_{\alpha}^{*} \in W_{2}^{1}\left(\omega, \begin{array}{c}
1 \\
x_{2}^{\kappa}
\end{array}\right) \subset W_{2}^{1}\left(\omega, \begin{array}{c}
1 \\
x_{2}^{2}
\end{array}\right) \quad \text { for } \quad 0<\kappa \leq 2 .
$$

Hence, in view of (3.10), Lemma 2.13, and the relations (4.13), (2.41), (2.33), we obtain

$$
\begin{aligned}
\int_{\omega}\left[\left(u_{1}^{*}\right)^{2}+\left(u_{2}^{*}\right)^{2}\right] d \omega & \leq 4 \int_{\omega} x_{2}^{2}\left[\left(u_{1,2}^{*}\right)^{2}+\left(u_{2,2}^{*}\right)^{2}\right] d \omega \\
& \leq 40 \int_{\omega} x_{2}^{2}\left[\left(u_{1,1}^{*}\right)^{2}+\left(u_{2,2}^{*}\right)^{2}+\left(u_{1,2}^{*}+u_{2,1}^{*}\right)^{2}\right] d \omega \\
& \leq 40 l^{2-\kappa} \int_{\omega} x_{2}^{\kappa}\left[\left(u_{1,1}^{*}\right)^{2}+\left(u_{2,2}^{*}\right)^{2}+\left(u_{1,2}^{*}+u_{2,1}^{*}\right)^{2}\right] d \omega \\
& \leq \frac{40 l^{2-\kappa}}{\mu h_{\kappa}} \int_{\omega} \mu h\left[\left(u_{1,1}^{*}\right)^{2}+\left(u_{2,2}^{*}\right)^{2}+\left(u_{1,2}^{*}+u_{2,1}^{*}\right)^{2}\right] d \omega .
\end{aligned}
$$

Let

$$
T_{0}:=\frac{40 l^{2-\kappa}}{\mu h_{\kappa}} ; \quad T_{\alpha}:=c^{2} \max _{\bar{\omega}}\left(p h^{\alpha}\right), \quad \alpha=1,2, \quad T_{3}:=T_{1} T_{0}+\frac{T_{2}}{6 \mu} .
$$

Taking into account (6.12), (1.10), from (6.11) we get

$$
\begin{aligned}
& \int_{\omega}\left[\left(u_{1}^{*}\right)^{2}+\left(u_{2}^{*}\right)^{2}\right] d \omega \\
& \leq T_{0} \mu \int_{\omega} h\left[2\left(u_{1,1}^{*}\right)^{2}+2\left(u_{2,2}^{*}\right)^{2}+\left(u_{1,2}^{*}+u_{2,1}^{*}\right)^{2}+18\left(v_{3}^{*}\right)^{2}\right] d \omega \\
& \leq T_{0} \int_{\omega} B\left(u^{*}, u^{*}\right) d \omega .
\end{aligned}
$$


From (2.11), (6.13), (1.11), (6.12), we have

$$
\begin{aligned}
\left\|u^{*}\right\|_{V_{h}^{0}}^{2}= & \int_{\omega}\left[\left(u_{1}^{*}\right)^{2}+\left(u_{2}^{*}\right)^{2}+B\left(u^{*}, u^{*}\right)\right] d \omega \leq\left(1+T_{0}\right) \int_{\omega} B\left(u^{*}, u^{*}\right) d \omega \\
= & \left(1+T_{0}\right) \int_{\omega}\left\{B_{c}\left(u^{*}, u^{*}\right)+c^{2} p\left[h\left(u_{1}^{*}\right)^{2}+h\left(u_{2}^{*}\right)^{2}+3 h^{3}\left(v_{3}^{*}\right)^{2}\right]\right\} d \omega \\
\leq & \left(1+T_{0}\right) \int_{\omega}\left\{B_{c}\left(u^{*}, u^{*}\right)+T_{1}\left[\left(u_{1}^{*}\right)^{2}+\left(u_{2}^{*}\right)^{2}\right]+\frac{T_{2}}{6 \mu} 18 \mu h\left(v_{3}^{*}\right)^{2}\right\} d \omega \\
\leq & \left(1+T_{0}\right) \int_{\omega}\left[B_{c}\left(u^{*}, u^{*}\right)+T_{1} T_{0} B\left(u^{*}, u^{*}\right)+\frac{T_{2}}{6 \mu} B\left(u^{*}, u^{*}\right)\right] d \omega \\
\leq & \left(1+T_{0}\right) \int_{\omega}\left\langle B_{c}\left(u^{*}, u^{*}\right)\right. \\
& \left.+T_{3}\left\{B_{c}\left(u^{*}, u^{*}\right)+c^{2} p\left[h\left(u_{1}^{*}\right)^{2}+h\left(u_{2}^{*}\right)^{2}+3 h^{3}\left(v_{3}^{*}\right)^{2}\right]\right\}\right\rangle d \omega \\
\leq & \left(1+T_{0}\right) \int_{\omega}\left\langle B_{c}\left(u^{*}, u^{*}\right)+T_{3}\left\{B_{c}\left(u^{*}, u^{*}\right)\right.\right. \\
& \left.\left.+T_{3}\left[B_{c}\left(u^{*}, u^{*}\right)+c^{2} p\left(h\left(u_{1}^{*}\right)^{2}+h\left(u_{2}^{*}\right)^{2}+3 h^{3}\left(v_{3}^{*}\right)^{2}\right)\right]\right\}\right\rangle d \omega \\
& (\text { repeating the same }(n-2)-\text { times more }) \\
\leq & \left(1+T_{0}\right) \int_{\omega}\left\{B_{c}\left(u^{*}, u^{*}\right) \frac{1-T_{3}^{n+1}}{1-T_{3}}\right. \\
& \left.+T_{3}^{n} c^{2} p\left[h\left(u_{1}^{*}\right)^{2}+h\left(u_{2}^{*}\right)^{2}+3 h^{3}\left(v_{3}^{*}\right)^{2}\right]\right\} d \omega .
\end{aligned}
$$

It is easy to see that, by virtue of (6.12),

$$
T_{3}=c^{2} \frac{240 l^{2-\kappa} \max _{\bar{\omega}}(p h)+h_{\kappa} \max _{\bar{\omega}}\left(p h^{2}\right)}{6 \mu h_{\kappa}} .
$$

From (6.2), (6.15) we obtain

$$
T_{3}<1
$$

In view of (6.16), for $n \rightarrow \infty$ in (6.14), we get

$$
\left\|u^{*}\right\|_{V_{h}^{0}}^{2} \leq \frac{1+T_{0}}{1-T_{3}} \int_{\omega} B_{c}\left(u^{*}, u^{*}\right) d \omega=\frac{1+T_{0}}{1-T_{3}} J_{c}\left(u^{*}, u^{*}\right),
$$

i.e.,

$$
J_{c}\left(u^{*}, u^{*}\right) \geq \frac{1-T_{3}}{1+T_{0}}\left\|u^{*}\right\|_{V_{h}^{0}}^{2} \quad \forall u^{*} \in V_{h}^{0} .
$$

Thus, the bilinear form $J_{c}\left(u^{*}, u^{*}\right)$ defined by $(5.20)$ is coercive. 
Now, let us show boundedness of $J_{c}\left(u^{*}, u^{*}\right)$ defined by $(5.20)$ in $V_{h}^{0}$. Indeed, from (5.20) with (1.10) for $u, u^{*} \in{ }^{B} W_{2, \overline{0}}^{1}$ we have

$$
\begin{aligned}
& \left|J_{c}\left(u, u^{*}\right)\right| \leq \int_{\omega}(\lambda h)^{\frac{1}{2}}\left|u_{1,1}+u_{2,2}+3 v_{3}\right|(\lambda h)^{\frac{1}{2}}\left|u_{1,1}^{*}+u_{2,2}^{*}+3 v_{3}^{*}\right| d \omega \\
& +\int_{\omega}(2 \mu h)^{\frac{1}{2}}\left|u_{1,1}\right|(2 \mu h)^{\frac{1}{2}}\left|u_{1,1}^{*}\right| d \omega+\int_{\omega}(2 \mu h)^{\frac{1}{2}}\left|u_{2,2}\right|(2 \mu h)^{\frac{1}{2}}\left|u_{2,2}^{*}\right| d \omega \\
& +\int_{\omega}(\mu h)^{\frac{1}{2}}\left|u_{2,1}+u_{1,2}\right|(\mu h)^{\frac{1}{2}}\left|u_{2,1}^{*}+u_{1,2}^{*}\right| d \omega+\int_{\omega}(18 \mu h)^{\frac{1}{2}}\left|v_{3}\right|(18 \mu h)^{\frac{1}{2}}\left|v_{3}^{*}\right| d \omega \\
& +\int_{\omega}\left(3 \mu h^{3}\right)^{\frac{1}{2}}\left|v_{3,1}\right|\left(3 \mu h^{3}\right)^{\frac{1}{2}}\left|v_{3,1}^{*}\right| d \omega+\int_{\omega}\left(3 \mu h^{3}\right)^{\frac{1}{2}}\left|v_{3,2}\right|\left(3 \mu h^{3}\right)^{\frac{1}{2}}\left|v_{3,2}^{*}\right| d \omega \\
& +T_{1} \int_{\omega}\left|u_{1}\right|\left|u_{1}^{*}\right| d \omega+T_{1} \int_{\omega}\left|u_{2}\right|\left|u_{2}^{*}\right| d \omega+\frac{T_{2}}{6 \mu} \int_{\omega}(18 \mu h)^{\frac{1}{2}}\left|v_{3}\right|(18 \mu h)^{\frac{1}{2}}\left|v_{3}^{*}\right| d \omega \\
& \leq\left[\int_{\omega} \lambda h\left(u_{1,1}+u_{2,2}+3 v_{3}\right)^{2} d \omega\right]^{\frac{1}{2}}\left[\int_{\omega} \lambda h\left(u_{1,1}^{*}+u_{2,2}^{*}+3 v_{3}^{*}\right)^{2} d \omega\right]^{\frac{1}{2}} \\
& +\left[\int_{\omega} 2 \mu h\left(u_{1,1}\right)^{2} d \omega\right]^{\frac{1}{2}}\left[\int_{\omega} 2 \mu h\left(u_{1,1}^{*}\right)^{2} d \omega\right]^{\frac{1}{2}} \\
& +\left[\int_{\omega} 2 \mu h\left(u_{2,2}\right)^{2} d \omega\right]^{\frac{1}{2}}\left[\int_{\omega} 2 \mu h\left(u_{2,2}^{*}\right)^{2} d \omega\right]^{\frac{1}{2}} \\
& +\left[\int_{\omega} \mu h\left(u_{2,1}+u_{1,2}\right)^{2} d \omega\right]^{\frac{1}{2}}\left[\int_{\omega} \mu h\left(u_{2,1}^{*}+u_{1,2}^{*}\right)^{2} d \omega\right] \\
& +\left[\int_{\omega} 18 \mu h\left(v_{3}\right)^{2} d \omega\right]\left[\int_{\omega} 18 \mu h\left(v_{3}^{*}\right)^{2} d \omega\right]^{\frac{1}{2}} \\
& +\left[\int_{\omega} 3 \mu h^{3}\left(v_{3,1}\right)^{2} d \omega\right]^{\frac{1}{2}}\left[\int_{\omega} 3 \mu h^{3}\left(v_{3,1}^{*}\right)^{2} d \omega\right]^{\frac{1}{2}} \\
& +\left[\int_{\omega} 3 \mu h^{3}\left(v_{3,2}\right)^{2} d \omega\right]^{\frac{1}{2}}\left[\int_{\omega} 3 \mu h^{3}\left(v_{3,2}^{*}\right)^{2} d \omega\right]^{\frac{1}{2}} \\
& +T_{1}\left[\int_{\omega}\left(u_{1}\right)^{2} d \omega\right]^{\frac{1}{2}}\left[\int_{\omega}\left(u_{1}^{*}\right)^{2} d \omega\right]^{\frac{1}{2}}+T_{1}\left[\int_{\omega}\left(u_{2}\right)^{2} d \omega\right]^{\frac{1}{2}}\left[\int_{\omega}\left(u_{2}^{*}\right)^{2} d \omega\right]^{\frac{1}{2}} \\
& +\frac{T_{2}}{6 \mu}\left[\int_{\omega} 18 \mu h\left(v_{3}\right)^{2} d \omega\right]^{\frac{1}{2}}\left[\int_{\omega} 18 \mu h\left(v_{3}^{*}\right)^{2} d \omega\right]^{\frac{1}{2}} \leq T_{4}\|u\|_{B} W_{2, \overline{0}}^{1}\left\|u^{*}\right\|_{B W_{2, \overline{0}}^{1}},
\end{aligned}
$$

where

$$
T_{4}=7+2 T_{1}+\frac{T_{2}}{6 \mu}
$$


In particular,

$$
\left|J_{c}\left(u, u^{*}\right)\right| \leq T_{4}\|u\|_{V_{h}^{0}}\left\|u^{*}\right\|_{V_{h}^{0}} \quad \forall u, u^{*} \in V_{h}^{0} .
$$

It is easy to see that the linear functional

$$
F_{c} u^{*}:=-\int_{\omega} f u^{*} d \omega-J_{c}\left(u^{0}, u^{*}\right), \quad u^{*} \in V_{h}^{0},
$$

is bounded in $V_{h}^{0}$. Indeed, because of (6.18) and

$$
\begin{aligned}
\left|\int_{\omega} f u^{*} d \omega\right| & \leq \sum_{\alpha=1}^{2}\left\|f_{\alpha}\right\|_{L_{2}(\omega)}\left\|u_{\alpha}^{*}\right\|_{L_{2}(\omega)}+\left\|(18 \mu h)^{-\frac{1}{2}} f_{3}\right\|_{L_{2}(\omega)}\left\|(18 \mu h)^{\frac{1}{2}} v_{3}^{*}\right\|_{L_{2}(\omega)} \\
& \leq\left(\sum_{\alpha=1}^{2}\left\|f_{\alpha}\right\|_{L_{2}(\omega)}+\left\|(18 \mu h)^{-\frac{1}{2}} f_{3}\right\|_{L_{2}(\omega)}\right)\left\|u^{*}\right\|_{V_{h}^{0}} \forall u^{*} \in V_{h}^{0},
\end{aligned}
$$

we have

$$
\left|F_{c} u^{*}\right| \leq\left[\sum_{\alpha=1}^{2}\left\|f_{\alpha}\right\|_{L_{2}(\omega)}+\left\|(18 \mu h)^{-\frac{1}{2}} f_{3}\right\|_{L_{2}(\omega)}+T_{4}\left\|u^{0}\right\|_{B} W_{2, \overline{0}}^{1}\right]\left\|u^{*}\right\|_{V_{h}^{0}} .
$$

According to the well-known Lax-Milgram theorem, by virtue of (6.17), (6.19), (6.21), there exists a unique $z \in V_{h}^{1}$ such that

$$
J_{c}\left(z, u^{*}\right)=F_{c} u^{*} \quad \forall u^{*} \in V_{h}^{0}
$$

and

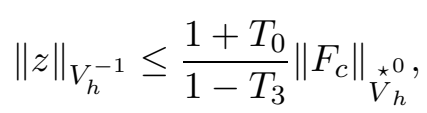

where $\stackrel{\star}{V}_{h}^{0}$ is dual to $V_{h}^{0}$.

From (6.20), (6.22) we get

$$
J_{c}\left(u, u^{*}\right)=-\int_{\omega} f u^{*} d \omega \quad \forall u^{*} \in V_{h}^{0},
$$

where

$$
u:=u^{0}+z \in{ }^{B} W_{2, \overline{0}}^{1}
$$

So,

$$
u-u^{0}=z \in V_{h}^{0} .
$$

Hence, (5.19) is fulfilled. Besides, (6.24) and (5.20) are the same, i.e., (5.20) holds as well. Thus, the existence and uniqueness of a weak solution is proved. We now show its continuous dependence on the data.

From (6.21) it is evident that

$$
\left\|F_{c}\right\|_{\substack{V_{h} \\ V^{0}}} \leq \sum_{\alpha=1}^{2}\left\|f_{\alpha}\right\|_{L_{2}(\omega)}+\left\|(18 \mu h)^{-\frac{1}{2}} f_{3}\right\|_{L_{2}(\omega)}+T_{4}\left\|u^{0}\right\|_{B} W_{2, \overline{0}}^{1} .
$$


In view of $(6.25),(6.23),(6.26)$, we have

$$
\begin{aligned}
\|u\|_{B} W_{2, \overline{0}}^{1} \leq & \left\|u^{0}\right\|_{B} W_{2, \overline{0}}^{1}+\|z\|_{V_{h}^{0}} \\
\leq & \frac{1+T_{0}}{1-T_{3}}\left[\sum_{\alpha=1}^{2}\left\|f_{\alpha}\right\|_{L_{2}(\omega)}+\left\|(18 \mu h)^{-\frac{1}{2}} f_{3}\right\|_{L_{2}(\omega)}\right] \\
& +\left(1+\frac{1+T_{0}}{1-T_{3}} T_{4}\right)\left\|u^{0}\right\|_{B} W_{2, \overline{0}}^{1}
\end{aligned}
$$

If we denote by

$$
C_{1}:=\max \left\{1+\frac{1+T_{0}}{1-T_{3}} T_{4}, \quad(18 \mu)^{-\frac{1}{2}} \frac{1+T_{0}}{1-T_{3}}\right\}
$$

since $T_{4} \geq 7$, from (6.27) we get (6.3).

Proof of Theorem 6.2: Let

$$
u^{*} \in V_{h}^{\kappa-2},
$$

then

$$
u_{\alpha}^{*} \in W_{2}^{1}\left(\omega, \begin{array}{c}
x_{2}^{\kappa-2} \\
h
\end{array}\right), \quad \alpha=1,2,
$$

and (6.9) is fulfilled. From (6.29), (2.69), and Lemma 2.13 we conclude

$$
u_{\alpha}^{*} \in \stackrel{\circ}{W_{2}}\left(\omega, \begin{array}{c}
x_{2}^{\kappa-2} \\
x_{2}^{\kappa}
\end{array}\right)
$$

Hence, in view of (3.3), (4.2), (2.33), we obtain

$$
\begin{aligned}
\int_{\omega} x_{2}^{\kappa-2}\left[\left(u_{1}^{*}\right)^{2}+\left(u_{2}^{*}\right)^{2}\right] d \omega & \leq \frac{4}{(\kappa-1)^{2}} \int_{\omega} x_{2}^{\kappa}\left[\left(u_{1,2}^{*}\right)^{2}+\left(u_{2,2}^{*}\right)^{2}\right] d \omega \\
& \leq \frac{4 C}{(\kappa-1)^{2}} \int_{\omega} x_{2}^{\kappa}\left[\left(u_{1,1}^{*}\right)^{2}+\left(u_{2,2}^{*}\right)^{2}+\left(u_{1,2}^{*}+u_{2,1}^{*}\right)^{2}\right] d \omega \\
& \leq \frac{4 C}{\mu h_{\kappa}(\kappa-1)^{2}} \int_{\omega} \mu h\left[\left(u_{1,1}^{*}\right)^{2}+\left(u_{2,2}^{*}\right)^{2}+\left(u_{1,2}^{*}+u_{2,1}^{*}\right)^{2}\right] d \omega .
\end{aligned}
$$

Let

$$
T^{0}:=\frac{4 C}{\mu h_{\kappa}(\kappa-1)^{2}} ; \quad T^{1}:=c^{2} \max _{\bar{\omega}}\left(x_{2}^{2-\kappa} p h\right), \quad T^{3}:=T^{1} T^{0}+\frac{T_{2}}{6 \mu} .
$$


Taking into account (6.32), (1.10), from (6.31) we get

$$
\begin{aligned}
& \int_{\omega} x_{2}^{\kappa-2}\left[\left(u_{1}^{*}\right)^{2}+\left(u_{2}^{*}\right)^{2}\right] d \omega \\
& \leq T^{0} \mu \int_{\omega} h\left[2\left(u_{1,1}^{*}\right)^{2}+2\left(u_{2,2}^{*}\right)^{2}+\left(u_{1,2}^{*}+u_{2,1}^{*}\right)^{2}+18\left(v_{3}^{*}\right)^{2}\right] d \omega \\
& \leq T^{0} \int_{\omega} B\left(u^{*}, u^{*}\right) d \omega .
\end{aligned}
$$

From (2.18), (2.11), (6.33), (1.11), (6.32), (6.12) we have

$$
\begin{aligned}
& \left\|u^{*}\right\|_{V_{h}^{\kappa-2}}^{2}=\int_{\omega}\left\{x_{2}^{\kappa-2}\left[\left(u_{1}^{*}\right)^{2}+\left(u_{2}^{*}\right)^{2}\right]+B\left(u^{*}, u^{*}\right)\right\} d \omega \leq\left(1+T^{0}\right) \int_{\omega} B\left(u^{*}, u^{*}\right) d \omega \\
& =\left(1+T^{0}\right) \int_{\omega}\left\{B_{c}\left(u^{*}, u^{*}\right)+c^{2} p\left[h\left(u_{1}^{*}\right)^{2}+h\left(u_{2}^{*}\right)^{2}+3 h^{3}\left(v_{3}^{*}\right)^{2}\right]\right\} d \omega \\
& \leq\left(1+T^{0}\right) \int_{\omega}\left\{B_{c}\left(u^{*}, u^{*}\right)+T^{1} x_{2}^{\kappa-2}\left[\left(u_{1}^{*}\right)^{2}+\left(u_{2}^{*}\right)^{2}\right]+\frac{T_{2}}{6 \mu} 18 \mu h\left(v_{3}^{*}\right)^{2}\right\} d \omega \\
& \leq\left(1+T^{0}\right) \int_{\omega}\left[B_{c}\left(u^{*}, u^{*}\right)+T^{1} T^{0} B\left(u^{*}, u^{*}\right)+\frac{T_{2}}{6 \mu} B\left(u^{*}, u^{*}\right)\right] d \omega \\
& \leq\left(1+T^{0}\right) \int_{\omega}\left\langle B_{c}\left(u^{*}, u^{*}\right)\right. \\
& \left.+T^{3}\left\{B_{c}\left(u^{*}, u^{*}\right)+c^{2} p\left[h\left(u_{1}^{*}\right)^{2}+h\left(u_{2}^{*}\right)^{2}+3 h^{3}\left(v_{3}^{*}\right)^{2}\right]\right\}\right\rangle d \omega \\
& \leq\left(1+T^{0}\right) \int_{\omega}\left\langle B_{c}\left(u^{*}, u^{*}\right)+T^{3}\left\{B_{c}\left(u^{*}, u^{*}\right)\right.\right. \\
& \left.\left.+T^{3}\left[B_{c}\left(u^{*}, u^{*}\right)+c^{2} p\left(h\left(u_{1}^{*}\right)^{2}+h\left(u_{2}^{*}\right)^{2}+3 h^{3}\left(v_{3}^{*}\right)^{2}\right)\right]\right\}\right\rangle d \omega \\
& \text { (repeating the same }(n-2)-\text { times more) } \\
& \leq\left(1+T^{0}\right) \int_{\omega}\left\{B_{c}\left(u^{*}, u^{*}\right) \frac{1-\left(T^{3}\right)^{n+1}}{1-T^{3}}\right. \\
& \left.+\left(T^{3}\right)^{n} c^{2} p\left[h\left(u_{1}^{*}\right)^{2}+h\left(u_{2}^{*}\right)^{2}+3 h^{3}\left(v_{3}^{*}\right)^{2}\right]\right\} d \omega .
\end{aligned}
$$

It is easy to see that, by virtue of $(6.32),(6.12)$,

$$
T^{3}=c^{2} \frac{24 C \max _{\bar{\omega}}\left(x_{2}^{2-\kappa} p h\right)+h_{\kappa}(\kappa-1)^{2} \max _{\bar{\omega}}\left(p h^{2}\right)}{6 \mu h_{\kappa}(\kappa-1)^{2}}
$$

From (6.5), (6.35) we obtain

$$
T^{3}<1
$$


In view of (6.36), for $n \rightarrow \infty$ in (6.34), we get

$$
\left\|u^{*}\right\|_{V_{h}^{\kappa-2}}^{2} \leq \frac{1+T^{0}}{1-T^{3}} \int_{\omega} B_{c}\left(u^{*}, u^{*}\right) d \omega=\frac{1+T^{0}}{1-T^{3}} J_{c}\left(u^{*}, u^{*}\right),
$$

i.e.,

$$
J_{c}\left(u^{*}, u^{*}\right) \geq \frac{1-T^{3}}{1+T^{0}}\left\|u^{*}\right\|_{V_{h}^{\kappa-2}}^{2} \quad \forall u^{*} \in V_{h}^{\kappa-2} .
$$

Thus, the bilinear form $J_{c}\left(u^{*}, u^{*}\right)$ is coercive.

Similarly to the proof of $(6.18)$, it is easy to show that

$$
\left|J_{c}\left(u, u^{*}\right)\right| \leq T^{4}\|u\|_{B W_{2, \overline{\kappa-2}}^{1}}\left\|u^{*}\right\|_{B W_{2, \overline{\kappa-2}}^{1}} \forall u, u^{*} \in{ }^{B} W_{2, \overline{\kappa-2}}^{1},
$$

where

$$
T^{4}:=7+2 T^{1}+\frac{T_{2}}{6 \mu}
$$

In particular,

$$
\left|J_{c}\left(u, u^{*}\right)\right| \leq T^{4}\|u\|_{V_{h}^{\kappa-2}}\left\|u^{*}\right\|_{V_{h}^{\kappa-2}} \quad \forall u, u^{*} \in V_{h}^{\kappa-2} .
$$

Now, let us show the boundedness of the following linear functional in $V_{h}^{\kappa-2}$

$$
F_{c} u^{*}:=-\int_{\omega} f u^{*} d \omega-J_{c}\left(u^{0}, u^{*}\right) .
$$

Indeed, because of (6.38) and

$$
\begin{aligned}
\left|\int_{\omega} f u^{*} d \omega\right| & =\left|\int_{\omega}\left[\sum_{\alpha=1}^{2} x_{2}^{\frac{2-\kappa}{2}} f_{\alpha} x_{2}^{\frac{\kappa-2}{2}} u_{\alpha}^{*}+(18 \mu h)^{-\frac{1}{2}} f_{3}(18 \mu h)^{\frac{1}{2}} v_{3}^{*}\right] d \omega\right| \\
& \leq\left[\sum_{\alpha=1}^{2}\left\|x_{2}^{\frac{2-\kappa}{2}} f_{\alpha}\right\|_{L_{2}(\omega)}+\left\|(18 \mu h)^{-\frac{1}{2}} f_{3}\right\|_{L_{2}(\omega)}\right]\left\|u^{*}\right\|_{V_{h}^{\kappa-2}} \forall u^{*} \in V_{h}^{\kappa-2},
\end{aligned}
$$

we have

$$
\begin{aligned}
\left|F_{c} u^{*}\right| & \leq\left[\sum_{\alpha=1}^{2}\left\|x_{2}^{\frac{2-\kappa}{2}} f_{\alpha}\right\|_{L_{2}(\omega)}+\left\|(18 \mu h)^{-\frac{1}{2}} f_{3}\right\|_{L_{2}(\omega)}\right. \\
& \left.+T^{4}\left\|u^{0}\right\|_{B} W_{2, \bar{\kappa}-2}^{1}\right]\left\|u^{*}\right\|_{V_{h}^{\kappa-2}} \forall u^{*} \in V_{h}^{\kappa-2} .
\end{aligned}
$$

According to the Lax-Milgram theorem, by virtue of (6.37), (6.39), (6.41), there exists a unique $z \in V_{h}^{\kappa-2}$ such that

$$
J_{c}\left(z, u^{*}\right)=F_{c} u^{*} \quad \forall u^{*} \in V_{h}^{\kappa-2}
$$

and

$$
\|z\|_{V_{h}^{\kappa-2}} \leq \frac{1+T^{0}}{1-T^{3}}\left\|F_{c}\right\|_{\substack{V_{h}^{\kappa-2} \\ V_{h}}}
$$


where $\stackrel{\star \star \kappa}{V}_{h}^{\kappa-2}$ is dual to $V_{h}^{\kappa-2}$.

From (6.40), (6.42) we get

$$
J_{c}\left(u, u^{*}\right)=-\int_{\omega} f u^{*} d \omega \quad \forall u^{*} \in V_{h}^{\kappa-2},
$$

where

$$
u:=u^{0}+z \in{ }^{B} W_{2, \overline{\kappa-2}}^{1}
$$

So,

$$
u-u^{0}=z \in V_{h}^{\kappa-2} .
$$

Hence, (5.24) is fulfilled. Besides, (6.44) and (5.25) are the same, i.e., (5.25) holds as well. Thus, the existence and uniqueness of a weak solution is proved. We show now its continuous dependence on the data.

From (6.41) it is evident that

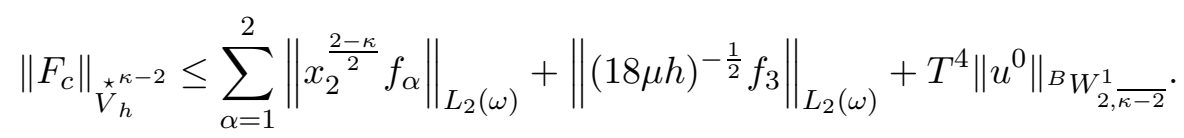

In view of $(6.45),(6.43),(6.46)$, we have

$$
\begin{aligned}
\|u\|_{B W_{2, \bar{\kappa}}^{1}} \leq & \left\|u^{0}\right\|_{B} W_{2, \overline{\kappa-2}}^{1}+\|z\|_{V_{h}^{\kappa-2}} \\
\leq & \frac{1+T^{0}}{1-T^{3}}\left[\sum_{\alpha=1}^{2}\left\|x_{2}^{\frac{2-\kappa}{2}} f_{\alpha}\right\|_{L_{2}(\omega)}+\left\|(18 \mu h)^{-\frac{1}{2}} f_{3}\right\|_{L_{2}(\omega)}\right] \\
& +\left(1+\frac{1+T^{0}}{1-T^{3}} T^{4}\right)\left\|u^{0}\right\|_{B W_{2, \kappa-2}^{1}} .
\end{aligned}
$$

Setting

$$
C_{2}:=\max \left\{1+\frac{1+T^{0}}{1-T^{3}} T^{4}, \quad(18 \mu)^{-\frac{1}{2}} \frac{1+T^{0}}{1-T^{3}}\right\},
$$

from (6.47) we get (6.6) because of $T^{4} \geq 7$.

\section{Some General Remarks}

Let us consider the static Problem 5.1 with homogeneous BCs (5.1), (5.4), (5.5). Obviously, for the $2 \mathrm{D}$ solution we have

$$
\stackrel{1}{v}:=\left(\stackrel{1}{v}_{10}, \stackrel{1}{v}_{20}, \stackrel{1}{v}_{31}\right) \in{ }^{B} W_{2, \overline{0}}^{1} \subset W_{2, \overline{0}}^{1} \subset W_{2, h}^{1} \quad \text { for } \quad 0<\kappa \leq 1 .
$$

On the other hand, as is shown in Jaiani, Kharibegeshvili, Natroshvili, Wendland, 2002, there exists a unique 2D solution

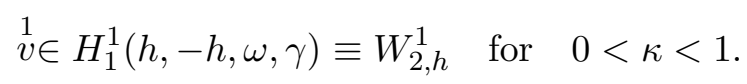


(for notation $H_{1}^{1}(h,-h, \omega, \gamma)$ see the above reference) with zero traces on $\Gamma$ and without any BCs on $\gamma_{0}$. But since 3D displacement vector $u \in H^{1}(\Omega)$, 3D surface force $F_{n}:=\left(X_{n 1}, X_{n 2}, X_{n 3}\right) \in L_{2}(\Omega)$, and using Fubini theorem, it is easy to show $\left.F_{n 0}\right|_{\gamma_{0}}=0,\left.F_{n 1}\right|_{\gamma_{0}}=0$. Thus, for the tension-compression problem $\left.T_{n}\right|_{\gamma_{0}}=0$.

So, both the 2D solutions coincide, and, therefore, correspond to the 3D BVP, when on the plate face surfaces (and if $0<\kappa<1$ on $\gamma_{0}$ as well) surface forces, i.e., stresses and on the non-cusped edge (lateral surface) zero displacements are given. It is remarkable that the static Problem 5.1 with BCs (5.1), (5.4), (5.5) we have solved for any $\kappa>1$ (for $\kappa \geq 2,{ }_{v}^{1} \in{ }^{B} W_{2, \overline{\kappa-2}}^{1} \subset W_{2, h}^{1} \equiv H_{1}^{1}(h,-h, \omega, \gamma$ ) in case of the tension-compression problem). Hence, the restriction $\kappa \leq 1$ in Jaiani, Kharibegashvili, Natroshvili, Wendland, 2002 was caused by the method of investigation used there, but a unique weak solution, as we have seen, exists for any $\kappa>0$, in particular, for $\kappa>1$ as well, in the same space $H_{1}^{1}(h,-h, \omega, \gamma)$, i.e., also in the case of a non-Lipschitz 3D domain $\Omega$.

Now, let us assume the existence of the above mixed 2D problem in the space $H_{N}^{1}(h,-h, \omega, \gamma)$ and construct a weak solution for the corresponding (above-mentioned) 3D problem in the case of a non-Lipschitz $\Omega$ as a limit in some sense for $N \rightarrow+\infty$. Such an approach seems to have a good chance for the proof of the existence theorem for a non-Lipschitz 3D domain which is an open problem up to now.

\section{References}

[1] R. L. Actis, B. A. Szabó, and Ch. Schwab, Hierarchic models for laminated plates and shells, Comput. Methods Appl. Mech. Engrg. 1999; 172(1-4): 79107.

[2] M. Avalishvili and D. Gordeziani, Investigation of two-dimensional models of elastic prismatic shell, Georgian Mathematical Journal, 2003; 10(1): 17-36.

[3] I. Babuška and L. Li, Hierarchic modeling of plates, Comput. \& Structures, 1991; 40: 419-430.

[4] I. Babuška and L. Li, The h-p-version of the finite element method in the plate modelling problem, Comm. Appl. Numer. Meth., 1992a; 8: 17-26.

[5] I. Babuška and L. Li, The problem of plate modelling: Theoretical and computational results, Comput. Meth. Appl. Mech. Engrg., 1992b; 100: 249-273.

[6] I. Babuška, B. A. Szabó and R. L. Actis, Hierarchic models for laminated composites, Internat. J. Numer. Methods Engrg., 1992; 33(3): 503-535.

[7] M. Dauge, E. Faou, and Z. Yosibash, Plates and shells: Asymptotic expansions and hierarchical models, Encyclopedia of Computational Mechanics. Edited by Erwin Stein, René de Borst and Thomas J.R. Hughes. John Wiley \& Sons, Ltd., 2004. 
[8] G. Devdariani, G. V. Jaiani, S. S. Kharibegashvili, and D. Natroshvili, The first boundary value problem for the system of cusped prismatic shells in the first approximation, Appl. Math. Inform., 2000; 5 (2): 26-46.

[9] G. Devdariani, The first boundary value problem for a degenerate elliptic system, Bull TICMI, 2001; 5: 23-24.

[10] D. G. Gordeziani, On the solvability of some boundary value problems for a variant of the theory of thin shells, Dokl. Akad. Nauk SSSR, 1974a; 215 (6): 1289-1292 (Russian).

[11] D. G. Gordeziani, To the exactness of one variant of the theory of thin shells, Dokl. Acad. Nauk SSSR, 1974b; 216 (4): 751-754 (Russian).

[12] V. Guliaev and V. Baganov and P. Lizunov, Nonclassic Theory of Shells, Vischa Shkola, Lviv, 1978 (Russian).

[13] G. V. Jaiani, Elastic bodies with non-smooth boundaries-cusped plates and shells, ZAMM-Zeitschrift für Angewandte Mathematik und Mechanik, 1996; 76 Suppl. 2: $117-120$.

[14] G. V. Jaiani, On a mathematical model of bars with variable rectangular crosssections, ZAMM-Zeitschrift für Angewandte Mathematik und Mechanik, 2001; 81, (3): 147-173.

[15] G. V. Jaiani, S. S. Kharibegashvili, D. G. Natroshvili, and W. L. Wendland, Hierarchical models for cusped plates, Preprint 2002/13. University of Stuttgart, Mathematical Institute A, Stuttgart, 2002.

[16] I. Khoma, The generalized theory of anisotropic shells, Naukova Dumka, Kiev, 1986 (Russian).

[17] A. R. Khvoles, The general representation for solutions of equilibrium equations of prismatic shell with variable thickness, Seminar of the Institute of Applied Mathematics of Tbilisi State University, Annot. of Reports, 1971; 5: 19-21 (Russian).

[18] A. Kufner and B. Opic. How to define reasonably weighted sobolev spaces, Commentations Matehamticae Universitatis Carolinae, 1984; 25 (3): 537-554.

[19] T. V. Meunargia, On nonlinear and nonshallow shells, Bulletin of TICMI, 1998, 2: $46-49$.

[20] S. M. Nikolskii, P. I. Lizorkin, and N. V. Miroshin, Weighted functional spaces and their applications to the investigation of boundary value problems for degenerate elliptic equations, Izvestia Vysshikh Uchebnykh Zavedenii, 1988; 8 (315): 4-30 (Russian).

[21] B. Opic and A. Kufner, Hardy-type inequalities, 1992. 
[22] Ch. Schwab, A-posteriori modeling error estimation for hierarchic plate models, Numer. Math., 1996; 74 (2): 221-259.

[23] B. Szabó and G. Sahrmann, Hierarchic plate and shell models based on pextension, Int. Jour. Numer. Meth. Engrg., 1988; 26: 1855-1881.

[24] T. S. Vashakmadze, The Theory of Anisotropic Plates, Kluwer Academic Publishers, Dordrecht-London-Boston, 1999.

[25] I. N. Vekua, On a method of computing prismatic shells, Akad. Nauk Gruzin. SSR. Trudy Tbiliss. Mat. Inst. Razmadze 1955; 21: 191-259 (Russian).

[26] I. N. Vekua, Theory of thin shallow shells of variable thickness, Akad. Nauk Gruzin. SSR Trudy Tbiliss. Mat. Inst. Razmadze, 1965; 30: 3-103 (Russian).

[27] I. N. Vekua, Shell theory: general methods of construction, Monographs, Advaced Texts and Surveys in Pure and Applied Mathematics, 25. Pitman (Advanced Publishing Program). Boston, MA. 1985.

[28] M. Vogelius and I. Babuška, On a dimensional reduction method. I. The optimal selection of basis functions, Math. Comp., 1981a; 37(155): 31-46.

[29] M. Vogelius and I. Babuška, On a dimensional reduction method. II. Some approximation-theoretic results, Math. Comp., 1981b; 37(155): 47-68.

[30] V. S. Zhgenti, To investigation of stress state of isotropic thick-walled shells of nonhomogeneous structure, Applied Mechanics, 1991; 27 (5): 37-44 (Russian).

E-mail addresses :

jaiani@viam.hepi.edu.ge

schulze@math.uni-potsdam.de 